\title{
DL-Lite with Temporalised Concepts, Rigid Axioms and Roles
}

\author{
A. Artale, ${ }^{1}$ R. Kontchakov, ${ }^{2}$ V. Ryzhikov, ${ }^{1}$ and M. Zakharyaschev ${ }^{2}$ \\ ${ }^{1}$ KRDB Research Centre \\ Free University of Bozen-Bolzano \\ I-39100 Bolzano, Italy \\ lastname@inf . unibz.it
${ }^{2}$ School of Comp. Science and Inf. Sys. Birkbeck College London WC1E 7HX, UK
$\{$ roman,michael\}@dcs.bbk.ac.uk

\begin{abstract}
We investigate the temporal extension of the description logic DL-Lite $e_{\text {bool }}^{(\mathcal{R N})}$ with the until operator on concepts, rigid (time-independent) and local (time-dependent) roles, and rigid TBox axioms. Using an embedding into the one-variable fragment of first-order temporal logic and the quasimodel technique, we prove that (i) the satisfiability problem for the resulting logic is PSPACE-complete, and that (ii) by weakening until to sometime in the future we obtain an NP-complete logic, which matches the complexities of the propositional linear-time temporal logics with the corresponding temporal operators.
\end{abstract}

\section{Introduction}

Numerous temporal extensions of various description logics (DLs) have been constructed and investigated since 1993, when K. Schild published his seminal paper [19]. (We refer the reader to the monograph [15] and survey papers $[3,9$, 17], where the history of the development of both interval- and point-based temporalised DLs is discussed in full detail.) There are various ways of introducing a temporal dimension in a DL. Temporal operators can be used as constructs for concepts, roles, TBox and ABox axioms - such concepts, roles or axioms are called temporalised. Alternatively, one may declare that a certain concept, role or axiom is rigid in the sense that its interpretation does not change in time. A number of complexity results have been obtained for different combinations of temporal operators and DLs. For instance, the following is known for combinations of $\mathcal{A L C}$ with the linear-time temporal logic $\mathcal{L} \mathcal{T} \mathcal{L}$ : the satisfiability problem for the temporal $\mathcal{A L C}$ is

- undecidable if temporalised concepts together with rigid axioms and roles are allowed in the language is enough); see [15] and references therein;

- 2ExpTime-complete if the language allows rigid concepts and roles with temporalised axioms [10];

- EXPSPACE-complete if the language allows temporalised concepts and axioms (but no rigid or temporalised roles) [15];

- ExPTIME-complete if the language allows only temporalised concepts and rigid axioms (but no rigid or temporalised roles) $[19,4]$. 
In other words, as long as one wants to express the temporal behaviour of only axioms and concepts (but not roles), the resulting combination is likely to be decidable. As soon as the combination allows reasoning about the temporal behaviour of binary relations, it becomes undecidable, unless we limit the means to describe the temporal behaviour of concepts. Furthermore, we notice that a better computational behaviour is exhibited in cases where rigid axioms are used instead of more general temporalised ones.

In this paper, we are interested in the scenario where axioms are rigid, concepts are temporalised and roles may be rigid or local (i.e., may change arbitrarily). To regain decidability in this case, one has to restrict either the temporal [8] or the DL component [7]. A decidable (in fact, 2ExPTIME-complete) logic $\mathbf{S} 5_{\mathcal{A L C} \mathcal{I}}[8]$ is obtained by combining the modal logic $\mathbf{S 5}$ with $\mathcal{A L C} \mathcal{L I}$. This approach weakens the temporal dimension to the much simpler S5, but can nevertheless represent rigid concepts and roles and allows one to state that concept and role memberships change in time (but without discriminating between changes in the past and future).

Temporal extensions of 'weak' DLs from the recently introduced DL-Lite and $\mathcal{E} \mathcal{L}$ families with rigid roles and temporalised axioms and concepts were investigated in [7]. It was shown that even in this case the resulting temporal DLs turn out to be very complex: ExPSPACE-complete for tractable $D L$-Lite $e_{\text {horn }}^{\mathcal{N}}$ and undecidable for tractable $\mathcal{E} \mathcal{L}$. An inspection of the lower bound proofs reveals, however, that they do not go through without the use of temporal and Boolean operators on TBox axioms. To find out the complexity of temporal DL-Lite logics without these constructs is the main aim of this paper.

Our most expressive DL-Lite logic DL-Lite $e_{\text {bool }}^{(\mathcal{R N})}[2]$ features non-qualified number restrictions and role inclusion axioms (with limited interaction), full Booleans on concepts as well as some other constructs. In $D L-L i t e_{\text {bool }}^{(\mathcal{R N})}$, the satisfiability problem is NP-complete for combined complexity, while instance checking is in $\mathrm{AC}^{0}$ for data complexity. We also consider the fragment $D L$-Lite core $_{(\mathcal{R N})}$ of $D L-L i t e_{b o o l}^{(\mathcal{R N})}$ with primitive concept inclusion axioms, for which satisfiability is NLOGSPACE-complete for combined complexity and answering positive existential queries is in $\mathrm{AC}^{0}$ for data complexity. (Because of this low data complexity of query answering, DL-Lite logics form the basis of OWL $2 \mathrm{QL}$, one of the three profiles of OWL 2; see http://www.w3.org/TR/ow12-profiles/.)

We consider two temporal extensions, $T_{\diamond} D L-L i t e_{\text {bool }}^{(\mathcal{R N})}$ and $T_{\mathcal{U}} D L-L i t e_{\text {bool }}^{(\mathcal{R N})}$, of $D L-$ Lite $_{\text {bool }}^{(\mathcal{R N})}$. Both logics weaken TDL-Lite ${ }_{b o o l}$ of [7] by allowing only rigid axioms; the temporalised concepts of $T_{\mathcal{U}} D L-L i t e_{b o o l}^{(\mathcal{R N})}$ can be built using temporal operators until $\mathcal{U}$, next-time $\bigcirc$ and their derivatives, while in $T_{\diamond} D L-L i t e_{\text {bool }}^{(\mathcal{R N})}$ they are limited to sometime in the future $\diamond$ and always in the future $\square$. We show that the satisfiability problem is NP-complete for $T_{\diamond} D L-L i t e_{b o o l}^{(\mathcal{R N})}$ and PSPACE-complete for $T_{\mathcal{U}} D L-L i t e_{\text {bool }}^{(\mathcal{R N})}$, which matches the complexity of the component logics. (Note, however, that they are not simple fusions of their components.) The lower bounds hold also for the core fragments of $T_{\diamond} D L-L i t e_{\text {bool }}^{(\mathcal{R N})}$ and $T_{\mathcal{U}} D L-$ Lite $_{\text {bool }}^{(\mathcal{R N})}$. 


\section{Temporal DL-Lite Logics}

We begin by defining temporal extensions $T_{\mathcal{U}} D L-L i t e_{\text {bool }}^{(\mathcal{R N})}$ and $T_{\diamond} D L-L i t e_{\text {bool }}^{(\mathcal{R N})}$ of the description logic $D L-L_{\text {ite }}^{(\mathcal{R N})}[1,2]$, which, in turn, extends the original $D L-$ Lite $_{\sqcap, \mathcal{F}}$ language [11-13] with full Booleans over concepts as well as cardinality restrictions on roles and role inclusion axioms with limited interaction.

The language of $T_{\mathcal{U}} D L$-Lite bool $_{(\mathcal{R N})}$ contains object names $a_{0}, a_{1}, \ldots$, concept names $A_{0}, A_{1}, \ldots$, local role names $P_{0}, P_{1}, \ldots$ and rigid role names $G_{0}, G_{1}, \ldots$; role names $S$, roles $R$, basic concepts $B$ and concepts $C$ are defined as follows:

$$
\begin{aligned}
& S \quad:=P_{i} \quad \mid G_{i}, \quad R \quad::=S \quad S^{-}, \\
& B \quad::=\perp\left|A_{i}\right| \geq q R, \\
& C \quad:=B|\neg C \quad| \quad C_{1} \sqcap C_{2}|\geq q R . C \quad| \quad C_{1} \mathcal{U} C_{2},
\end{aligned}
$$

where $q \geq 1$ is a natural number. The language $T_{\diamond} D L-L i t e_{b o o l}^{(\mathcal{R N})}$ is a proper sub-language of $T_{\mathcal{U}} D L-$ Lite $_{\text {bool }}^{(\mathcal{R N})}$ in which the until operator $\mathcal{U}$ can occur only in concepts of the form $\top \mathcal{U} C$, where $\top=\neg \perp$. As usual in temporal logic, we denote $\top \mathcal{U} C$ by $\diamond C$, and also write $\square C$ for $\neg \diamond \neg C$ and $\bigcirc C$ for $\perp \mathcal{U} C$ (so, $\bigcirc$ is a $T_{\mathcal{U}} D L-L i t e_{\text {bool }}^{(\mathcal{R N})}$ concept construct). Other standard abbreviations we use are as follows: $C_{1} \sqcup C_{2}=\neg\left(\neg C_{1} \sqcap \neg C_{2}\right), \exists R=(\geq 1 R)$ and $\leq q R=\neg(\geq q+1 R)$.

A $T_{\mathcal{U}} D L-$ Lite ${ }_{\text {bool }}^{(\mathcal{R N})}$ TBox $\mathcal{T}$ is a finite set of concept inclusions, role inclusions, and role disjointness, irreflexivity and reflexivity constraints of the form:

$$
C_{1} \sqsubseteq C_{2}, \quad R_{1} \sqsubseteq R_{2}, \quad \operatorname{Dis}\left(R_{1}, R_{2}\right), \quad \operatorname{Irr}(S) \quad \text { and } \quad \operatorname{Ref}(S) .
$$

We write $i n v(R)$ for $S^{-}$if $R=S$, and for $S$ if $R=S^{-}$. Denote by $\sqsubseteq_{\mathcal{T}}^{*}$ the reflexive and transitive closure of $\left\{\left(R, R^{\prime}\right),\left(\operatorname{inv}(R), \operatorname{inv}\left(R^{\prime}\right)\right) \mid R \sqsubseteq R^{\prime} \in \mathcal{T}\right\}$. Say that $R^{\prime}$ is a proper sub-role of $R$ in $\mathcal{T}$ if $R^{\prime} \sqsubseteq_{\mathcal{T}}^{*} R$ and $R \Xi_{\mathcal{T}}^{*} R^{\prime}$. The following syntactic conditions, limiting the interaction between number restrictions and role inclusions, are imposed on $T_{\mathcal{U}} D L-L_{\text {Lite }}^{(\mathcal{R N})}$ bool TBoxes $\mathcal{T}$ (cf. [18, 2]):

(inter) if $R$ has a proper sub-role in $\mathcal{T}$ then $\mathcal{T}$ contains no negative occurrences ${ }^{1}$ of number restrictions $\geq q R$ or $\geq q \operatorname{inv}(R)$ with $q \geq 2$;

(exists) $\mathcal{T}$ may contain only positive occurrences of $\geq q R . C$, and if $\geq q R . C$ occurs in $\mathcal{T}$ then $\mathcal{T}$ does not contain negative occurrences of $\geq q^{\prime} R$ or $\geq q^{\prime} \operatorname{inv}(R)$, for $q^{\prime} \geq 2$.

It follows that no TBox can contain both a functionality constraint $\geq 2 R \sqsubseteq \perp$ and an occurrence of $\geq q R$.C, for some $q \geq 1$ and some role $R$. (These conditions are required for NP-completeness of satisfiability in DL-Lite ${ }_{\text {bool }}^{(\mathcal{R N})}$.)

An $A B o x \mathcal{A}$ consists of assertions of the form:

$$
\bigcirc^{n} B(a), \quad \square B(a), \quad \bigcirc^{n} S(a, b), \quad \square S(a, b), \quad \bigcirc^{n} \neg S(a, b) \quad \text { and } \quad \square \neg S(a, b),
$$

\footnotetext{
${ }^{1}$ An occurrence of a concept on the right-hand (left-hand) side of a concept inclusion is called negative if it is in the scope of an odd (even) number of negations $\neg$; otherwise the occurrence is called positive.
} 
where $B$ is a basic concept, $S$ a (local or rigid) role name, $a, b$ object names and $\bigcirc^{n}$ denotes the sequence of $n$ next-time operators $\bigcirc$, for $n \geq 0$ (inverse roles could also be allowed in the ABoxes, but they are just syntactic sugar). The TBox and ABox together form the knowledge base $(\mathrm{KB}) \mathcal{K}=(\mathcal{T}, \mathcal{A})$.

A $T_{\mathcal{U}} D L$-Lite ${ }_{\text {bool }}^{(\mathcal{R N})}$ interpretation $\mathcal{I}$ is a function on natural numbers $\mathbb{N}$ :

$$
\mathcal{I}(n)=\left(\Delta^{\mathcal{I}}, a_{0}^{\mathcal{I}}, \ldots, A_{0}^{\mathcal{I}(n)}, \ldots, P_{0}^{\mathcal{I}(n)}, \ldots, G_{0}^{\mathcal{I}(n)}, \ldots\right),
$$

where $\Delta^{\mathcal{I}}$ is a non-empty set, the domain of $\mathcal{I}, a_{i}^{\mathcal{I}} \in \Delta^{\mathcal{I}}, A_{i}^{\mathcal{I}(n)} \subseteq \Delta^{\mathcal{I}}$ and $P_{i}^{\mathcal{I}(n)}, G_{i}^{\mathcal{I}(n)} \subseteq \Delta^{\mathcal{I}} \times \Delta^{\mathcal{I}}$, for all $i$ and all $n \in \mathbb{N}$. Furthermore, $a_{i}^{\mathcal{I}} \neq a_{j}^{\mathcal{I}}$ for $i \neq j$ (which means that we adopt the unique name Assumption) and $G_{i}^{\mathcal{I}(n)}=G_{i}^{\mathcal{I}(m)}$, for all $n, m \in \mathbb{N}$. The role and concept constructs are interpreted in $\mathcal{I}$ as follows: for each moment of time $n \in \mathbb{N}$,

$$
\begin{array}{rlrl}
\left(S^{-}\right)^{\mathcal{I}(n)} & =\left\{(y, x) \in \Delta^{\mathcal{I}} \times \Delta^{\mathcal{I}} \mid(x, y) \in S^{\mathcal{I}(n)}\right\}, & \perp^{\mathcal{I}(n)}=\emptyset, \\
(\geq q R . C)^{\mathcal{I}(n)} & =\left\{x \in \Delta^{\mathcal{I}} \mid \sharp\left\{y \in C^{\mathcal{I}} \mid(x, y) \in R^{\mathcal{I}(n)}\right\} \geq q\right\}, \quad(\neg C)^{\mathcal{I}(n)}=\Delta^{\mathcal{I}} \backslash C^{\mathcal{I}(n)}, \\
(C \sqcap D)^{\mathcal{I}(n)} & =C^{\mathcal{I}(n)} \cap D^{\mathcal{I}(n)}, & (\geq q R)^{\mathcal{I}(n)}=(\geq q R . \neg \perp)^{\mathcal{I}(n)}, \\
(C \mathcal{U} D)^{\mathcal{I}(n)} & =\bigcup_{k>n}\left(D^{\mathcal{I}(k)} \cap \bigcap_{n<m<k} C^{\mathcal{I}(m)}\right), &
\end{array}
$$

where $\sharp X$ is the cardinality of $X$. The satisfaction relation $\models$ is defined as follows:

$$
\begin{gathered}
\mathcal{I} \models C_{1} \sqsubseteq C_{2} \quad \text { iff } C_{1}^{\mathcal{I}(n)} \subseteq C_{2}^{\mathcal{I}(n)} \text { for all } n \geq 0, \\
\mathcal{I}=R_{1} \sqsubseteq R_{2} \quad \text { iff } R_{1}^{\mathcal{I}(n)} \subseteq R_{2}^{\mathcal{I}(n)} \text { for all } n \geq 0, \\
\mathcal{I} \models \operatorname{Dis}\left(R_{1}, R_{2}\right) \quad \text { iff } \forall n \geq 0\left(R_{1}^{\mathcal{I}(n)} \cap R_{2}^{\mathcal{I}(n)}=\emptyset\right) \quad\left(R_{1} \text { and } R_{2} \text { are disjoint }\right), \\
\mathcal{I} \models \operatorname{Irr}(S) \quad \text { iff } \forall x \in \Delta^{\mathcal{I}} \forall n \geq 0(x, x) \notin S^{\mathcal{I}(n)} \quad(S \text { is irreflexive }), \\
\mathcal{I} \models \operatorname{Ref}(S) \quad \text { iff } \forall x \in \Delta^{\mathcal{I}} \forall n \geq 0(x, x) \in S^{\mathcal{I}(n)} \quad(S \text { is reflexive }), \\
\mathcal{I} \models \bigcirc^{n} B(a) \text { iff } a^{\mathcal{I}} \in B^{\mathcal{I}(n)}, \quad \mathcal{I} \models \square B(a) \text { iff } \forall n>0 a^{\mathcal{I}} \in B^{\mathcal{I}(n)}, \\
\mathcal{I} \models \bigcirc^{n} S(a, b) \text { iff }\left(a^{\mathcal{I}}, b^{\mathcal{I}}\right) \in S^{\mathcal{I}(n)}, \quad \mathcal{I} \models \square S(a, b) \text { iff } \forall n>0\left(a^{\mathcal{I}}, b^{\mathcal{I}}\right) \in S^{\mathcal{I}(n)}, \\
\mathcal{I} \models \bigcirc^{n} \neg S(a, b) \text { iff }\left(a^{\mathcal{I}}, b^{\mathcal{I}}\right) \notin S^{\mathcal{I}(n)}, \quad \mathcal{I} \models \square \neg S(a, b) \text { iff } \forall n>0\left(a^{\mathcal{I}}, b^{\mathcal{I}}\right) \notin S^{\mathcal{I}(n)} .
\end{gathered}
$$

We say that $\mathcal{I}$ is a model of a $\mathrm{KB} \mathcal{K}$ if $\mathcal{I}=\alpha$ for all $\alpha$ in $\mathcal{K}$; in this case we also write $\mathcal{I} \models \mathcal{K}$. A concept $A$ (role $R$ ) is satisfiable w.r.t. $\mathcal{K}$ if there are a model $\mathcal{I}$ of $\mathcal{K}$ and $n \geq 0$ such that $A^{\mathcal{I}(n)} \neq \emptyset\left(R^{\mathcal{I}(n)} \neq \emptyset\right)$. Note that role symmetry $\operatorname{Sym}(S)$ and asymmetry $\operatorname{Asym}(S)$ constraints are syntactic sugar in this language: they can be equivalently replaced with $S^{-} \sqsubseteq S$ and $\operatorname{Dis}\left(S, S^{-}\right)$, respectively.

It should be noted that $T_{\mathcal{U}} D L-L i t e_{\text {bool }}^{(\mathcal{R} \mathcal{N})}$ is not a simple fusion of DL-Lite ${ }_{\text {bool }}^{(\mathcal{R N})}$ and $\mathcal{L} \mathcal{T} \mathcal{L}$. Indeed, consider $\mathcal{K}=\left(\left\{\diamond \exists R^{-} \sqsubseteq \perp, \exists R \sqsubseteq \diamond \exists R\right\},\{\exists R(a)\}\right)$. Clearly, $\mathcal{K}$ is not satisfiable in $T_{\mathcal{U}} D L-L i t e_{\text {bool }}^{(\mathcal{R N})}$. However, it is satisfiable both in $D L-L i t e_{\text {bool }}^{(\mathcal{R N})}$ (if we substitute the temporal concepts by fresh $D L$-Lite ${ }_{\text {bool }}^{(\mathcal{R N})}$ concepts) and in $\mathcal{L} \mathcal{T} \mathcal{L}$ (by substituting $\exists R$ concepts with fresh atomic propositions).

\section{$3 \quad T_{\mathcal{U}} D L-$ Lite $_{\text {bool }}^{(\mathcal{R N})}$ and First-Order Temporal Logic}

For a $T_{\mathcal{U}} D L-L i t e_{\text {bool }}^{(\mathcal{R N})} \mathrm{KB} \mathcal{K}=(\mathcal{T}, \mathcal{A})$, let $o b(\mathcal{A})$ be the set of all object names occurring in $\mathcal{A}$. Let role ${ }^{ \pm}(\mathcal{K})$ be the set of rigid and local role names, together 
with their inverses, occurring in $\mathcal{K}$ and grole $^{ \pm}(\mathcal{K})$ its subset of rigid roles. For $R \in \operatorname{role}^{ \pm}(\mathcal{K})$, let $Q_{\mathcal{K}}^{R}$ be the set of natural numbers containing 1 and all the numerical parameters $q$ for which $\geq q R$ or $\geq q R$. $C$ occurs in $\mathcal{K}$.

With every object name $a \in o b(\mathcal{A})$ we associate the individual constant $a$ of $\mathcal{Q T} \mathcal{L}^{1}$, the one-variable fragment of first-order temporal logic over $(\mathbb{N},<)$, and with every concept name $A$ the unary predicate $A(x)$ from the signature of $\mathcal{Q T} \mathcal{L}^{1}$. For each $R \in \operatorname{role}^{ \pm}(\mathcal{K})$, we also introduce $\left|Q_{\mathcal{K}}^{R}\right|$ fresh unary predicates $E_{q} R(x)$, for $q \in Q_{\mathcal{K}}^{R}$. Intuitively, for each $n \geq 0, E_{1} R(x)$ and $E_{1} R^{-}(x)$ represent the domain and range of $R$ at moment $n$ (i.e., $E_{1} R(x)$ and $E_{1} R^{-}(x)$ are interpreted by the sets of points with at least one $R$-successor and at least one $R$-predecessor at moment $n$, respectively), while $E_{q} R(x)$ and $E_{q} R^{-}(x)$ represent the sets of points with at least $q$ distinct $R$-successors and at least $q$ distinct $R$-predecessors at moment $n$.

Let us consider first the sub-language of $T_{\mathcal{U}} D L-L i t e_{\text {bool }}^{(\mathcal{R N})}$ without qualified number restrictions and role constraints; we denote it by $T_{\mathcal{U}} D L-L i t e_{\text {bool }}^{(\mathcal{R N N})^{-}}$. Without loss of generality, we will assume that $Q_{\mathcal{K}}^{R} \subseteq Q_{\mathcal{K}}^{R^{\prime}}$ whenever $R \sqsubseteq_{\mathcal{T}}^{*} R^{\prime}$ (for if this is not the case we can always add the missing numbers to $Q_{\mathcal{K}}^{R^{\prime}}$ by introducing fictitious concept inclusions of the form $\left.\perp \sqsubseteq \geq q R^{\prime}\right)$. By induction on the construction of a $T_{\mathcal{U}} D L-L_{i t e}^{(\mathcal{R N})^{-}}{ }^{-}$concept $C$ we define the $\mathcal{Q T} \mathcal{L}^{1}$ - formula $C^{*}$ :

$$
\begin{aligned}
\perp^{*} & =\perp, & (A)^{*} & =A(x), \\
(\geq q R)^{*} & =E_{q} R(x), & (\neg C)^{*} & =\neg C^{*}(x), \\
\left(C_{1} \sqcap C_{2}\right)^{*} & =C_{1}^{*}(x) \wedge C_{2}^{*}(x), & \left(C_{1} \mathcal{U} C_{2}\right)^{*} & =C_{1}^{*}(x) \mathcal{U} C_{2}^{*}(x),
\end{aligned}
$$

and then extend this translation to $T_{\mathcal{U}} D L-L i t e_{\text {bool }}^{(\mathcal{R N})^{-}}$TBoxes $\mathcal{T}$ by taking:

$$
\left.\mathcal{T}^{*}=\bigwedge_{C_{1} \sqsubseteq C_{2} \in \mathcal{T}} \square^{+} \forall x\left(C_{1}^{*}(x) \rightarrow C_{2}^{*}(x)\right)\right) \bigwedge_{\substack{R \sqsubseteq R^{\prime} \in \mathcal{T} \text { or } \\ i n v(R) \sqsubseteq i n v\left(R^{\prime}\right) \in \mathcal{T}}} \bigwedge_{q \in Q_{\mathcal{K}}^{R}} \square^{+} \forall x\left(E_{q} R(x) \rightarrow E_{q} R^{\prime}(x)\right),
$$

where $\square^{+} \varphi=\varphi \wedge \square \varphi$. For $R \in \operatorname{role}^{ \pm}(\mathcal{K})$, we need two $\mathcal{Q} \mathcal{T} \mathcal{L}^{1}$-sentences:

$$
\begin{aligned}
\varepsilon_{R} & =\exists x E_{1} R(x) \rightarrow \exists x \operatorname{inv}\left(E_{1} R\right)(x), \\
\delta_{R} & =\bigwedge_{\substack{q, q^{\prime} \in Q_{\mathcal{K}}^{R}, q^{\prime}>q \\
q^{\prime}>q^{\prime \prime}>q \text { for no } q^{\prime \prime} \in Q_{\mathcal{K}}^{R}}} \forall x\left(E_{q^{\prime}} R(x) \rightarrow E_{q} R(x)\right),
\end{aligned}
$$

where $\operatorname{inv}\left(E_{1} R\right)$ is the predicate $E_{1} S^{-}(x)$ if $R=S$ and $E_{1} S(x)$ if $R=S^{-}$, for a role name $S$. Sentence (1) says that if the domain of $R$ is non-empty then its range is non-empty either; the meaning of (2) should be obvious.

Now we define 'temporal slices' of the ABox $\mathcal{A}^{2}$. Denote by $N_{\mathcal{A}}$ the maximum $n$ with $\bigcirc^{n} B(a) \in \mathcal{A}, \bigcirc^{n} S(a, b) \in \mathcal{A}$ or $\bigcirc^{n} \neg S(a, b) \in \mathcal{A}$ (or 0 if there are no such

\footnotetext{
${ }^{2}$ We slightly abuse notation and, for $R \in \operatorname{role}^{ \pm}(\mathcal{K})$, write $\bigcirc^{n} R\left(a_{i}, a_{j}\right) \in \mathcal{A}$ to indicate that $\bigcirc^{n} S\left(a_{i}, a_{j}\right) \in \mathcal{A}$ if $R=S$, or $\bigcirc^{n} S\left(a_{j}, a_{i}\right) \in \mathcal{A}$ if $R=S^{-}$, where $S$ is a (local or rigid) role name; similarly for $\square R\left(a_{i}, a_{j}\right) \in \mathcal{A}$.
} 
assertions in $\mathcal{A})$. For a rigid role $R \in$ role $^{ \pm}(\mathcal{K})$, we take:

$$
\mathcal{A}_{R}^{\square}=\left\{R(a, b) \mid \bigcirc^{n} R^{\prime}(a, b) \in \mathcal{A} \text { or } \square R^{\prime}(a, b) \in \mathcal{A} \text {, and } R^{\prime} \sqsubseteq_{\mathcal{T}}^{*} R\right\}
$$

and $\mathcal{A}_{R}^{n}=\mathcal{A}_{R}^{\square}$, for all $n, 0 \leq n \leq N_{\mathcal{A}}$. For a local role $R \in$ role $^{ \pm}(\mathcal{K})$, we take:

$$
\begin{aligned}
& \mathcal{A}_{R}^{\square}=\left\{R(a, b) \mid \square R^{\prime}(a, b) \in \mathcal{A}, \text { for } R^{\prime} \sqsubseteq_{\mathcal{T}}^{*} R\right\} \\
& \cup\left\{R(a, b) \mid R^{\prime}(a, b) \in \mathcal{A}_{R^{\prime}}^{\square} \text { for } R^{\prime} \sqsubseteq_{\mathcal{T}}^{*} R\right\}, \\
& \mathcal{A}_{R}^{n}=\left\{R(a, b) \mid \bigcirc^{n} R^{\prime}(a, b) \in \mathcal{A}, \text { for } R^{\prime} \sqsubseteq_{\mathcal{T}}^{*} R\right\} \\
& \cup\left\{R(a, b) \mid R^{\prime}(a, b) \in \mathcal{A}_{R^{\prime}}^{\square} \text { for } R^{\prime} \sqsubseteq_{\mathcal{T}}^{*} R\right. \\
&\text { and either } \left.R^{\prime} \in \text { grole }^{ \pm}(\mathcal{K}) \text { or } n>0\right\} .
\end{aligned}
$$

We also set $\mathcal{A}^{\square}=\bigcup_{R \in \text { role }}^{ \pm(\mathcal{K})} A_{R}^{\square}$ and $\mathcal{A}^{n}=\bigcup_{R \in \text { role }^{ \pm}(\mathcal{K})} A_{R}^{n}$, for $0 \leq n \leq N_{\mathcal{A}}$. The $\mathcal{Q T} \mathcal{L}^{1}$ translation of the ABox $\mathcal{A}$ is defined as follows:

$$
\begin{aligned}
\mathcal{A}^{*}=\bigwedge_{\bigcirc^{n} B(a) \in \mathcal{A}} \bigcirc^{n} B^{*}(a) & \wedge \bigwedge_{R(a, b) \in \mathcal{A}^{n}} \bigcirc^{n} E_{q_{R, a, \mathcal{A}^{n}}} R(a) \wedge \bigwedge_{R(a, b) \in \mathcal{A}^{\square}} \square E_{q_{R, a, \mathcal{A}^{\square}}} R(a) \\
& \wedge \bigwedge_{\bigcirc^{n} \neg S(a, b) \in \mathcal{A}}\left(\bigcirc^{n} \neg S(a, b)\right)^{\perp} \wedge \bigwedge_{\square \neg S(a, b) \in \mathcal{A}}(\square \neg S(a, b))^{\perp},
\end{aligned}
$$

where, for a role $R$, an $\mathrm{ABox} \mathcal{A}^{\prime}$ and $a \in o b\left(\mathcal{A}^{\prime}\right)$,

$q_{R, a, \mathcal{A}^{\prime}}=\max \left(\{0\} \cup\left\{q \in Q_{\mathcal{K}}^{R} \mid R\left(a, a_{i}\right) \in \mathcal{A}^{\prime}, 1 \leq i \leq q \& a_{i_{1}} \neq a_{i_{2}}\right.\right.$ if $\left.\left.i_{1} \neq i_{2}\right\}\right)$,

and $\left(\bigcirc^{n} \neg S(a, b)\right)^{\perp}=\perp$ if $S(a, b) \in \mathcal{A}^{n}$ and $\top$ otherwise, and $(\square \neg S(a, b))^{\perp}=\perp$ if $S(a, b) \in \mathcal{A}^{\square}$ or $S(a, b) \in \mathcal{A}^{n}$, for $0<n \leq N_{\mathcal{A}}$, and $T$ otherwise. Finally, let

$$
\mathcal{K}^{\ddagger}=\mathcal{T}^{*} \wedge \bigwedge_{R \in \text { role }^{ \pm}(\mathcal{K})} \square^{+}\left(\varepsilon_{R} \wedge \delta_{R}\right) \wedge \bigwedge_{T \in \text { grole }}(\mathcal{K}) \bigwedge_{q \in Q_{\mathcal{K}}^{T}} \square^{+} \forall x\left(E_{q} T(x) \leftrightarrow \square E_{q} T(x)\right) \wedge \mathcal{A}^{*} .
$$

Observe that the length of $\mathcal{K}^{\ddagger}$ is linear in length of $\mathcal{K}$. It can be shown in a way similar to [7, Theorem 2 and Corollary 3]) that we have:

Lemma 1. A $T_{\mathcal{U}} D L-$ Lite $_{\text {bool }}^{(\mathcal{R N})^{-}} K B \mathcal{K}=(\mathcal{T}, \mathcal{A})$ is satisfiable iff the $\mathcal{Q T} \mathcal{L}^{1}$ sentence $\mathcal{K}^{\ddagger}$ is satisfiable.

Proof. $(\Leftarrow)$ Let $\mathfrak{M}$ be a first-order temporal model with a countable domain $D$ and let $(\mathfrak{M}, 0) \models \mathcal{K}^{\ddagger}$ (if $\mathcal{K}^{\ddagger}$ is satisfiable then such an $\mathfrak{M}$ clearly exists). We denote the interpretations of unary predicates $P$ in $\mathfrak{M}$ at moment $n$ by $P^{\mathfrak{M}, n}$ and the interpretations of constants $a$ in $\mathfrak{M}$ by $a^{\mathfrak{M}}$ (without loss of generality we assume that the $a^{\mathfrak{M}}$ are all distinct). We are going to construct a model $\mathcal{I}$ of $\mathcal{K}$ based on the domain $\Delta=\bigcup_{m=0}^{\infty} W_{m}$, where

$$
W_{0}=\left\{a^{\mathfrak{M}} \mid a \in o b(\mathcal{A})\right\} \subseteq D \quad \text { and } \quad W_{m+1}=W_{m} \cup(D \times \mathbb{N} \times\{m\}) .
$$

The interpretations of object names in $\mathcal{I}$ are given by their interpretations in $\mathfrak{M}: a^{\mathcal{I}(n)}=a^{\mathfrak{M}} \in W_{0}$. The interpretations $A^{\mathcal{I}(n)}$ of concept names $A$ in $\mathcal{I}$ are 
set to be $A^{\mathcal{I}(n)}=\left\{w \in \Delta \mid(\mathfrak{M}, n) \models A^{*}[c p(w)]\right\}$, where $c p: \Delta \rightarrow D$ is defined by taking:

$$
c p(w)= \begin{cases}w, & \text { if } w \in W_{0}, \\ d, & \text { if } w=(d, k, m) \in D \times \mathbb{N} \times \mathbb{N} .\end{cases}
$$

We call $w$ a copy of $c p(w)$.

It remains to define $S^{\mathcal{I}(n)}$ for each role $S$ in $\mathcal{K}$ and $n \in \mathbb{N}$. Let us first consider a minimal role $S$ for which $R \sqsubseteq_{\mathcal{T}}^{*} S$ implies $S \sqsubseteq_{\mathcal{T}}^{*} R$, for every $R \in$ role $^{ \pm}(\mathcal{K})$. Let $[S]=\left\{R \in \operatorname{role}^{ \pm}(\mathcal{K}) \mid R \sqsubseteq_{\mathcal{T}}^{*} S\right.$ and $\left.S \sqsubseteq_{\mathcal{T}}^{*} R\right\}$. Consider first the case when $[S] \cap$ grole $^{ \pm}(\mathcal{K})=\emptyset$ (i.e., $S$ is not equivalent to a rigid role). Fix some $n \in \mathbb{N}$. We set $S^{\mathcal{I}(n)}=\bigcup_{m=0}^{\infty} S^{n, m}$, where $S^{n, m} \subseteq W_{m} \times W_{m}$ are defined inductively (on $m \geq 0$ ) as follows. For the basis of induction, set

$$
S^{n, 0}=\left\{\left(a^{\mathfrak{M}}, b^{\mathfrak{M}}\right) \in W_{0} \times W_{0} \mid S(a, b) \in \mathcal{A}^{n}, n \leq N_{\mathcal{A}} \text {, or } S(a, b) \in \mathcal{A}^{\square}, n>0\right\} .
$$

Suppose the $S^{n, m}$ have been defined. Given $R=S$ or $S^{-}$, the required $R$-rank $r^{n}(R, d)$ of $d \in D$ at moment $n$ is $\max \left(\{0\} \cup\left\{q \in Q_{\mathcal{K}}^{R} \mid(\mathfrak{M}, n) \models E_{q} R[d]\right\}\right)$. By (2), if $r^{n}(R, d)=q$ then, for every $q^{\prime} \in Q_{\mathcal{K}}^{R}$, we have $(\mathfrak{M}, n) \models E_{q^{\prime}} R[d]$ whenever $q^{\prime} \leq q$ and $(\mathfrak{M}, n) \models \neg E_{q^{\prime}} R[d]$ whenever $q<q^{\prime}$. The actual $R$-rank $r_{m}^{n}(R, w)$ of $w \in \Delta$ at moment $n$ and step $m$ is $\sharp\left\{w^{\prime} \in W_{m} \mid\left(w, w^{\prime}\right) \in R^{n, m}\right\}$, where $R^{n, m}=S^{n, m}$ if $R=S$ and $R^{n, m}=\left\{\left(w, w^{\prime}\right) \mid\left(w^{\prime}, w\right) \in S^{n, m}\right\}$ if $R=S^{-}$. It will follow from our construction that $r_{m}^{n}(S, w) \leq r^{n}(S, c p(w))$, for all $w \in W_{m}$ (we leave the easy inductive proof to the reader). Consider now the two sets of defects $\Lambda_{S}^{n, m}$ and $\Lambda_{S^{-}}^{n, m}$ in $S^{n, m}$, where

$$
\Lambda_{R}^{n, m}=\left\{w \in W_{m} \mid r_{m}^{n}(R, w)<r^{n}(R, c p(w))\right\} .
$$

The purpose of $\Lambda_{R}^{n, m}$ is to identify those 'defective' points $w \in W_{m}$ from which precisely $r^{n}(R, c p(w))$ distinct $R$-arrows should start (according to $\mathfrak{M}$ ), but some arrows are missing (only $r_{m}^{n}(R, w)$ many arrows exist). To 'cure' these defects, we need a pool $F_{m} \subseteq W_{m+1} \backslash W_{m}$ of witnesses that can be used at step $m$ of the unravelling construction: more precisely, it contains, for each role $R$, a countably infinite supply of points $w$ (witnesses for $R$ ) such that $c p(w)=d$ and $(\mathfrak{M}, n) \models i n v\left(E_{1} R\right)[d]$, provided that $(\mathfrak{M}, n) \models \exists x\left(E_{1} R(x) \vee i n v\left(E_{1} R\right)(x)\right)$ (by (1), either both $E_{1} R(x)$ and $i n v\left(E_{1} R\right)(x)$ are empty or both are non-empty). It should be emphasised that this set will be the same for all roles $R$ and all moments of time $n$. We extend $S^{n, m}$ to $S^{n, m+1}$ according to the following rules:

$\left(\Lambda_{S}^{n, m}\right)$ If $w \in \Lambda_{S}^{n, m}$, then let $q=r^{n}(S, d)-r_{m}^{n}(S, w)$ and $d=c p(w)$. We have $(\mathfrak{M}, n) \models E_{q^{\prime}} S[d]$ for some $q^{\prime} \in Q_{\mathcal{K}}^{S}$. By $(2),(\mathfrak{M}, n) \models E_{1} S[d]$. In this case we take $q$ fresh witnesses $w_{1}, \ldots, w_{q} \in F_{m}$ for $S$, remove them from $F_{m}$ and then add the pairs $\left(w, w_{i}\right), 1 \leq i \leq q$, to $S^{n, m+1}$; we also add the pairs $\left(w_{i}, w\right), 1 \leq i \leq q$, to $S^{n, m+1}$ if $S^{-} \in[S]$.

$\left(\Lambda_{S^{-}}^{n, m}\right)$ The mirror image of $\left(\Lambda_{S}^{n, m}\right)$.

In a way similar to [2, Section 5.3] one can show that, for all $n \geq 0$ and $R=S, S^{-}$

$$
(\mathfrak{M}, n) \models E_{q} R[c p(w)] \quad \text { iff } \quad w \in(\geq q R)^{\mathcal{I}(n)} .
$$


Once we have defined $S^{\mathcal{I}(n)}$ for $S$, we set $\left(S^{\prime}\right)^{\mathcal{I}(n)}=S^{\mathcal{I}(n)}$ for all $S^{\prime} \in[S]$ and $n \geq 0$ (observe that $\mathcal{A}_{S}^{\square}=\mathcal{A}_{S^{\prime}}^{\square}$ and $\mathcal{A}_{S}^{n}=\mathcal{A}_{S^{\prime}}^{n}$, for all $n$, if $S^{\prime} \in[S]$ ). If $[S] \cap \operatorname{grole}^{ \pm}(\mathcal{K}) \neq \emptyset$, then we perform the above unravelling procedure at moment 0 and set $S^{\mathcal{I}(n)}=S^{\mathcal{I}(0)}$ for all $n>0$, and $\left(S^{\prime}\right)^{\mathcal{I}(n)}=S^{\mathcal{I}(n)}$, for all $S^{\prime} \in[S]$ and $n \geq 0$ (observe that $\mathcal{A}_{S}^{\square}=\mathcal{A}_{S^{\prime}}^{\square}=\mathcal{A}_{S}^{n}=\mathcal{A}_{S^{\prime}}^{n}$, for all $n$, if $S^{\prime} \in[S]$ ).

Suppose now that $S$ has a proper sub-role and we have already defined the $R^{\mathcal{I}(n)}$ for all such proper sub-roles $R$. For $S$ with $[S] \cap \operatorname{grol}^{ \pm}(\mathcal{K})=\emptyset$, the unravelling procedure is analogous to the one described above: the basis of induction is defined as above; then, at every step, we first expand $S^{n, m}$ with the pairs that belong to its proper sub-roles, i.e., $\bigcup_{R \sqsubseteq_{T}^{*} S, S \Xi_{\mathcal{T}}^{*} R} R^{n, m}$, and only after that start curing the defects (remember that a point in $F_{m}$ can never be used twice as a witness). As the actual rank may be greater than the required rank, we have the following: for all $n \geq 0$ and $R=S, S^{-}$,

$$
\text { if }(\mathfrak{M}, n) \models E_{q} R[c p(w)] \quad \text { then } \quad w \in(\geq q R)^{\mathcal{I}(n)} \text {. }
$$

If $[S] \cap \operatorname{grole}^{ \pm}(\mathcal{K}) \neq \emptyset$ then we perform the unravelling procedure only for the moment 0 and, at each step, first expand $S^{0, m}$ with the pairs that belong to its proper sub-roles at any moment of time (i.e., $\bigcup_{R \sqsubseteq_{\mathcal{T}}^{*} S, S \Xi_{\mathcal{T}}^{*} R} \bigcup_{n=0}^{\infty} R^{n, m}$ ) and only after that cure the defects (yet again a point in $F_{m}$ can never be used twice as a witness). Finally, we set $S^{\mathcal{I}(n)}=S^{\mathcal{I}(0)}$, for all $n>0$, and $\left(S^{\prime}\right)^{\mathcal{I}(n)}=S^{\mathcal{I}(n)}$, for all $S^{\prime} \in[S]$ and $n \geq 0$.

It remains to show that the constructed interpretation $\mathcal{I}$ is indeed a model of $\mathcal{K}$. It follows from the construction that $\mathcal{I} \models R_{1} \sqsubseteq R_{2}$, for each $R_{1} \sqsubseteq R_{2} \in \mathcal{T}$. It also follows that (3) holds for every role without proper sub-roles and, for a role that has proper sub-roles, (4) is enough in view of (inter): by induction on the structure of concepts, one can show that, for each concept inclusion $C_{1} \sqsubseteq C_{2} \in \mathcal{T}$, we have $\mathcal{I} \models C_{1} \sqsubseteq C_{2}$ whenever $(\mathfrak{M}, n) \models \forall x\left(C_{1}^{*}(x) \rightarrow C_{2}^{*}(x)\right)$, for all $n \geq 0$. Thus, $\mathcal{I} \models \mathcal{T}$. We also have $\mathcal{I} \models \mathcal{A}$ and thus $\mathcal{I} \models \mathcal{K}$.

For $(\Rightarrow)$ we refer the reader to [7, Theorem 2 and Corollary 3$]$.

Denote by $T_{\mathcal{U}}^{0} D L-L i t e_{\text {bool }}^{\mathcal{N}}$ the fragment of $T_{\mathcal{U}} D L-L i t e_{\text {bool }}^{(\mathcal{R N})^{-}}$such that (i) it has no rigid roles, (ii) its TBoxes contain only concept inclusions and (iii) its ABoxes contain only assertions of the form $\bigcirc^{n} B(a)$ and $\square B(a)$.

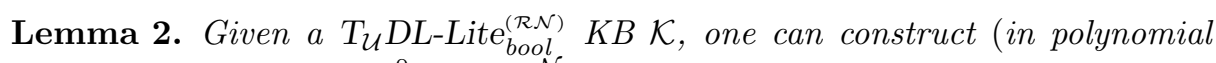
time) an equisatisfiable $T_{\mathcal{U}}^{0} D L$-Lite $e_{\text {bool }}^{\mathcal{N}} K B \mathcal{K}^{\prime}$.

Proof. As a first step, one can construct a $T_{\mathcal{U}} D L-L i t e_{\text {bool }}^{(\mathcal{R N})^{-}} \mathrm{KB}$ such that it is equisatisfiable with $\mathcal{K}$. The proof is based on the forest model property (cf. [2, Remark 5.15]) and is similar to that of [2, Lemma 5.17]. It shows how to get rid of qualified number restrictions, role disjointness, reflexivity and irreflexivity constraints. So, without loss of generality we may assume that $\mathcal{K}$ is a $T_{\mathcal{U}}$ DL-Lite bool $^{(\mathcal{R N})^{-}}$KB.

Next, we set $\mathcal{K}^{\prime}=(\{\top \sqsubseteq \perp\}, \emptyset)$ if $\bigcirc^{n} \neg S(a, b) \in \mathcal{A}$ and $S(a, b) \in \mathcal{A}^{n}$, or $\square \neg S(a, b) \in \mathcal{A}$ and $S(a, b) \in \mathcal{A}^{\square}$ or $S(a, b) \in \mathcal{A}^{n}, 0<n \leq N_{\mathcal{A}}$. Otherwise, let $\mathcal{A}_{0}$ be the part of $\mathcal{A}$ with assertions of the form $\bigcirc^{n} B(a)$ and $\square B(a)$ only 
and let $\mathcal{T}_{0}$ be the part of $\mathcal{T}$ that contains no role inclusion axioms. Consider $\mathcal{K}^{\prime}=\left(\mathcal{T}_{0} \cup \mathcal{T}^{\prime \prime}, \mathcal{A}_{0} \cup \mathcal{A}^{\prime \prime}\right)$, where

$$
\begin{aligned}
\mathcal{T}^{\prime \prime}= & \left\{\square(\geq q T) \sqsubseteq(\geq q T),(\geq q T) \sqsubseteq \square(\geq q T) \mid q \in Q_{\mathcal{K}}^{T}, T \in \text { grole }^{ \pm}(\mathcal{K})\right\} \\
& \cup\left\{(\geq q R) \sqsubseteq\left(\geq q R^{\prime}\right) \mid q \in Q_{\mathcal{K}}^{R}, R \sqsubseteq R^{\prime} \in \mathcal{T} \text { or } \operatorname{inv}(R) \sqsubseteq \operatorname{inv}\left(R^{\prime}\right) \in \mathcal{T}\right\}, \\
\mathcal{A}^{\prime \prime}= & \left\{\bigcirc^{n}\left(\geq q_{R, a, \mathcal{A}^{n}} R\right)(a) \mid R(a, b) \in \mathcal{A}^{n}\right\} \cup\left\{\square\left(\geq q_{R, a, \mathcal{A}^{\square}} R\right)(a) \mid R(a, b) \in \mathcal{A}^{\square}\right\} .
\end{aligned}
$$

Clearly, $\mathcal{K}^{\ddagger}=\left(\mathcal{K}^{\prime}\right)^{\ddagger}$. The claim follows immediately from Lemma 1 .

\section{Satisfiability of $T_{\mathcal{U}} D L-L i t e_{b o o l}^{(\mathcal{R N})} \mathrm{KBs}$ is PSpace-complete}

It follows from Lemma 2 that satisfiability of $T_{\mathcal{U}} D L-L i t e_{\text {bool }}^{(\mathcal{R N})} \mathrm{KBs}$ is reducible to satisfiability of $T_{\mathcal{U}}^{0} D L-L i t e_{\text {bool }}^{\mathcal{N}}$ KBs. Our plan is as follows. First, we define a notion of quasimodel for a $T_{\mathcal{U}}^{0} D L-L i t e_{\text {bool }}^{\mathcal{N}} \mathrm{KB}$ and prove that such a $\mathrm{KB}$ is satisfiable iff there exists a quasimodel for it. Then we show that if there is a quasimodel for a KB then there exists an ultimately periodic quasimodel such that both the length of the prefix and the length of the period are exponential in the length of the KB. The existence of such a quasimodel can be checked in non-deterministic polynomial space, which together with Lemma 2 provides us with a PSPACE upper complexity bound for satisfiability in $T_{\mathcal{U}} D L-L i t e_{\text {bool }}^{(\mathcal{R N})}$; the matching lower bound follows from the complexity of $\mathcal{L} \mathcal{T} \mathcal{L}$.

Let $\mathcal{K}=(\mathcal{T}, \mathcal{A})$ be a $T_{\mathcal{U}}^{0} D L$-Lite bool $_{\text {bol }}^{\mathcal{N}} \mathrm{KB}$. We assume $o b(\mathcal{A}) \neq \emptyset$. Denote by $e v(\mathcal{K})$ the set of all concepts of the form $C \mathcal{U} D$ occurring in $\mathcal{K}$. We introduce, for every $C \mathcal{U} D \in e v(\mathcal{K})$, a fresh concept name $F_{C \mathcal{U} D}$, the surrogate of $C \mathcal{U} D$, and then, for a concept $C$, denote by $\bar{C}$ the result of replacing each $C^{\prime} \mathcal{U} D^{\prime}$ in $C$, which is not in the scope of another $\mathcal{U}$, with the surrogate $F_{C^{\prime} \mathcal{U} D^{\prime}}$. For a $T_{\mathcal{U}}^{0} D L-L i t e_{\text {bool }}^{\mathcal{N}}$ TBox $\mathcal{T}$, denote by $\overline{\mathcal{T}}$ the $D L$-Lite $\mathrm{V}_{\text {bool }}^{\mathcal{N}}$ TBox obtained by replacing every concept $C$ in $\mathcal{T}$ with $\bar{C}$.

Let $c l(\mathcal{K})$ be the closure under negation of all concepts occurring in $\mathcal{T}$ together with the $\exists R$, for $R \in \operatorname{role}^{ \pm}(\mathcal{K})$, and the $B$, for $\bigcirc^{n} B(a) \in \mathcal{A}$ or $\square B(a) \in \mathcal{A}$. A type for $\mathcal{K}$ is a subset $\mathbf{t}$ of $\operatorname{cl}(\mathcal{K})$ such that

- $C \sqcap D \in \mathbf{t}$ iff $C, D \in \mathbf{t}$, for every $C \sqcap D \in \operatorname{cl}(\mathcal{K})$;

$-\neg C \in \mathbf{t}$ iff $C \notin \mathbf{t}$, for every $C \in \operatorname{cl}(\mathcal{K})$.

A type $\mathbf{t}$ for $\mathcal{K}$ is realisable if the concept $\Pi_{C \in \mathbf{t}} \bar{C}$ is satisfiable w.r.t. $\overline{\mathcal{T}}$.

A function $r$ mapping $\mathbb{N}$ to types for $\mathcal{K}$ is called a coherent and saturated run for $\mathcal{K}$ if the following conditions are satisfied:

(real) $r(i)$ is realisable, for every $i \geq 0$,

(coh) for all $0 \leq j<i$ and $C \mathcal{U} D \in e v(\mathcal{K})$, if $D \in r(i)$ and $C \in r(k)$, for all $k$, $j<k<i$, then $C \mathcal{U} D \in r(j)$;

(sat) for all $i \geq 0$ and $C \mathcal{U} D \in e v(\mathcal{K})$, if $C \mathcal{U} D \in r(i)$ then there is $j>i$ such that $D \in r(j)$ and $C \in r(k)$ for all $k, i<k<j$. 
Given a model $\mathcal{I}$ of $\mathcal{K}$ and $d \in \Delta^{\mathcal{I}}$, let $\operatorname{run}_{\mathcal{I}}(d): i \mapsto\left\{C \in \operatorname{cl}(\mathcal{K}) \mid d \in C^{\mathcal{I}(i)}\right\}$. Clearly, each $\operatorname{run}_{\mathcal{I}}(d)$ is a coherent and saturated run for $\mathcal{K}$. For a run $r$ and a finite sequence $s=(s(0), \ldots, s(n))$ of types for $\mathcal{K}$, we denote by $s^{k}$ the $k$ repetitions of $s$ and let

$$
\begin{aligned}
& r^{<i}=(r(0), \ldots, r(i-1)), \quad s^{\omega}=(s(0), \ldots, s(n), s(0), \ldots, s(n), \ldots), \\
& r^{\geq i}=(r(i), r(i+1), \ldots), \quad s \cdot r=(s(0), \ldots, s(n), r(0), r(1), \ldots) .
\end{aligned}
$$

A witness for $\mathcal{K}$ is a pair of the form $(r, \Xi)$, where $r$ is a coherent and saturated run for $\mathcal{K}, \Xi \subseteq \mathbb{N}$ and $|\Xi| \leq 1$. A quasimodel for $\mathcal{K}$ is a quadruple $\mathfrak{Q}=\left\langle W, K, K_{0}, L\right\rangle$, where $W$ is a set of witnesses for $\mathcal{K}$ and $K, K_{0}, L$ are natural numbers with $0 \leq K<K_{0}<L$ such that:

(run) $W=\left\{\left(r_{a}, \emptyset\right) \mid a \in o b(\mathcal{A})\right\} \cup\left\{\left(r_{R},\left\{i_{R}\right\}\right) \mid R \in \Omega\right\}$, for some $\Omega \subseteq \operatorname{role}^{ \pm}(\mathcal{K})$; (rep) $r_{R}(K)=r_{R}\left(K_{0}\right)$, for each $\left(r_{R},\left\{i_{R}\right\}\right) \in W$;

(obj) if $\bigcirc^{n} B(a) \in \mathcal{A}$ then $B \in r_{a}(n)$; if $\square B(a) \in \mathcal{A}$ then $B \in r_{a}(i)$ for all $i>0$; (role) for all $i \geq 0$ and $R \in \operatorname{role}^{ \pm}(\mathcal{K})$, if $\exists R^{-} \in r(i)$, for some $(r, \Xi) \in W$, then $\left(r_{R},\left\{i_{R}\right\}\right) \in W, \exists R \in r_{R}\left(i_{R}\right)$ and either $i \leq i_{R}<K$ or $K \leq i_{R}<L$.

Theorem 1. $A T_{\mathcal{U}}^{0} D L-$ Lite $_{\text {bool }}^{\mathcal{N}} K B \mathcal{K}$ is satisfiable iff there is a quasimodel $\mathfrak{Q}=$ $\left\langle W, K, K_{0}, L\right\rangle$ for $\mathcal{K}$ with $L \leq N_{\mathcal{A}}+2^{|c l(\mathcal{K})| \cdot \mid \text { role }^{ \pm}(\mathcal{K}) \mid} \cdot\left(\mid\right.$ role $\left.^{ \pm}(\mathcal{K}) \mid+1\right)+2^{|c l(\mathcal{K})|}+2$.

Proof. Suppose $\mathcal{I} \models \mathcal{K}$. For $m \geq 0$, let

$$
\mathbf{F}^{m}=\left\{R \in \operatorname{role}^{ \pm}(\mathcal{K}) \mid \text { there is } i \geq m \text { with } R^{\mathcal{I}(i)} \neq \emptyset\right\} .
$$

Lemma 3. For all $n, v \geq 0$, there exists $m$ such that $n \leq m \leq n+v \cdot\left|\mathbf{F}^{0}\right|$ and, for every role $R \in \mathbf{F}^{0}$, either $R \in \mathbf{F}^{m+v+1}$ or $R \notin \mathbf{F}^{m+1}$.

Proof. If a role $R$ is non-empty infinitely often then $R \in \mathbf{F}^{m+v+1}$, for any $m$. So we have to consider only those roles that are non-empty finitely many times. Let $\mathbf{F G}=\left\{R \in \operatorname{role}^{ \pm}(\mathcal{K}) \mid\right.$ there is $i \geq 0$ with $\left.R \notin \mathbf{F}^{i}\right\}$. For $R \in \mathbf{F G} \cap \mathbf{F}^{0}$, let $i_{R}=\min \left\{i \mid R \notin \mathbf{F}^{i+1}\right\}$ (i.e., $i_{R}$ is the last moment when $R$ is non-empty). If $\max \left\{i_{R} \mid R \in \mathbf{F G}\right\} \leq n+v \cdot\left|\mathbf{F}^{0}\right|$, we take $m=\max \left(\{n\} \cup\left\{i_{R} \mid R \in \mathbf{F G}\right\}\right)$. Clearly, FG $\cap \mathbf{F}^{m+1}=\emptyset$ (so all roles in $\mathbf{F G}$ are empty after $m$ ). Otherwise, $\mathbf{F G} \cap \mathbf{F}^{0} \neq \emptyset$ and without loss of generality we may assume that $\mathbf{F G} \cap \mathbf{F}^{0}=$ $\left\{R_{1}, \ldots, R_{s}\right\}$ and $i_{R_{1}} \leq i_{R_{2}} \leq \cdots \leq i_{R_{s}}$. If $i_{R_{1}}>n+v$, we take $m=n$; then $\mathbf{F G} \cap \mathbf{F}^{0} \subseteq \mathbf{F}^{m+v+1}$ (all roles in $\mathbf{F G} \cap \mathbf{F}^{0}$ are non-empty after $m+v$ ). Otherwise, $i_{R_{1}} \leq n+v$ and $i_{R_{s}}>n+v \cdot\left|\mathbf{F}^{0}\right|$, whence $i_{R_{s}}-i_{R_{1}}>(v-1) \cdot\left|\mathbf{F}^{0}\right|$. Let $j_{0}$ be the smallest $j, 1 \leq j<s$, such that $i_{R_{j}} \geq n$ and $i_{R_{j+1}}-i_{R_{j}}>v$ (it exists as $\left.s \leq\left|\mathbf{F}^{0}\right|\right)$, and let $m=i_{R_{j_{0}}}$. We then clearly have $R_{1}, \ldots, R_{j_{0}} \notin \mathbf{F}^{m+1}$ and $R_{j_{0}+1}, \ldots, R_{s} \in \mathbf{F}^{m+v+1}$.

Let $V=2^{|c l(\mathcal{K})| \cdot\left|r o l e^{ \pm}(\mathcal{K})\right|}$. By Lemma 3, there is $M, N_{\mathcal{A}} \leq M \leq N_{\mathcal{A}}+V \cdot\left|\mathbf{F}^{0}\right|$, such that, for every role $R \in \mathbf{F}^{0}$, either $R \in \mathbf{F}^{M+V+1}$ or $R \notin \mathbf{F}^{M+1}$. We set

$$
i_{R}= \begin{cases}\min \left\{i \geq M+V+1 \mid R^{\mathcal{I}(i)} \neq \emptyset\right\}, & R \in \mathbf{F}^{M+V+1}, \\ \max \left\{i \mid R^{\mathcal{I}(i)} \neq \emptyset\right\}, & R \in \mathbf{F}^{0} \backslash \mathbf{F}^{M+1} .\end{cases}
$$


Clearly, for each $R \in \mathbf{F}^{0}$, either $i_{R} \leq M$ or $i_{R} \geq M+V+1$. We fix some $d_{R} \in(\exists R)^{\mathcal{I}\left(i_{R}\right)}$ and set $r_{R}=\operatorname{run}_{\mathcal{I}}\left(d_{R}\right)$, for each $R \in \mathbf{F}^{0}$. For $a \in o b(\mathcal{A})$, set $r_{a}=\operatorname{run}_{\mathcal{I}}\left(a^{\mathcal{I}}\right)$. Let

$$
W=\left\{\left(r_{a}, \emptyset\right) \mid a \in o b(\mathcal{A})\right\} \cup\left\{\left(r_{R},\left\{i_{R}\right\}\right) \mid R \in \mathbf{F}^{0}\right\} .
$$

Clearly, (run) and (obj) hold for $W$. Also, $\exists R^{-} \in r(i)$ iff $\exists R \in r_{R}\left(i_{R}\right)$ and $\left(r_{R},\left\{i_{R}\right\}\right) \in W$, for all $(r, \Xi) \in W$ and $i \geq 0$.

Observe that there are $K, K_{0}$ such that $M<K<K_{0} \leq M+V+1$ and $r_{R}(K)=r_{R}\left(K_{0}\right)$, for all $\left(r_{R},\left\{i_{R}\right\}\right) \in W$. Let $L=K_{0}+T+1$. Next, for each $\left(r_{R},\left\{i_{R}\right\}\right) \in W$ with $i_{R} \geq L$, we construct a new witness $\left(r_{R}^{\prime},\left\{i_{R}^{\prime}\right\}\right)$ such that $\exists R \in r_{R}^{\prime}\left(i_{R}^{\prime}\right)$ and $i_{R}^{\prime}<L$ : we remove every part $\left(r_{R}(n), \ldots, r_{R}\left(n^{\prime}\right)\right)$ of the run $r_{R}$ such that $r_{R}(n)=r_{R}\left(n^{\prime}+1\right)$, for $K_{0}<n<n^{\prime}<i_{R}$. Let $\left(r_{R}^{\prime},\left\{i_{R}^{\prime}\right\}\right)$ be the result of this operation. It should be clear that $r_{R}^{\prime}$ is a coherent and saturated run for $\mathcal{K}$ and, as there are only $T$ different types for $\mathcal{K}$, we have $i_{R}^{\prime}<L$. Denote by $W^{\prime}$ the resulting set of witnesses. It is as an exercise for the reader to check that $\mathfrak{Q}=\left\langle W^{\prime}, K, K_{0}, L\right\rangle$ is a quasimodel for $\mathcal{K}$.

$(\Leftarrow)$ Let $\mathfrak{Q}=\left\langle W, K, K_{0}, L\right\rangle$ be a quasimodel for $\mathcal{K}$. We construct a model for $\mathcal{K}^{\ddagger}$ which, by Theorem 1 , will show that $\mathcal{K}$ is satisfiable. Let

$$
\begin{aligned}
& \mathfrak{R}=\left\{r_{a} \mid\left(r_{a}, \emptyset\right) \in W\right\} \cup \\
& \left\{\left(r_{R}^{<K} \cdot\left(r_{R}(K), \ldots, r_{R}\left(K_{0}-1\right)\right)^{i} \cdot r_{R}^{\geq K_{0}}\right)^{\geq j} \mid\left(r_{R},\left\{i_{R}\right\}\right) \in W, i>0, j \geq 0\right\} .
\end{aligned}
$$

Clearly, each $r \in \mathfrak{R}$ is a coherent and saturated run for $\mathcal{K}$. Moreover, if we have $\left(r_{R},\left\{i_{R}\right\}\right) \in W$ and $i_{R}<K$ then, for all $i, 0 \leq i \leq i_{R}$, there is $r^{\prime} \in \Re$ with $\exists R \in r^{\prime}(i)$. And if $\left(r_{R},\left\{i_{R}\right\}\right) \in W$ and $i_{R} \geq K$ then, for all $i \geq 0$, there is $r^{\prime} \in \mathfrak{R}$ with $\exists R \in r^{\prime}(i)$. As follows from (role), for each $R \in \Omega$, we have $R^{-} \in \Omega$ and either $i_{R} \geq K$ and $i_{R^{-}} \geq K$ or $i_{R}=i_{R^{-}}<K$. So, for all $i \geq 0$ and $r \in \Re$,

$$
\text { if } \exists R^{-} \in r(i) \text { then there is } r^{\prime} \in \Re \text { such that } \exists R \in r^{\prime}(i) \text {. }
$$

We construct a first-order temporal model $\mathfrak{M}$ based on the domain $D=\mathfrak{R}$ by taking $a^{\mathfrak{M}}=r_{a}$, for each $a \in o b(\mathcal{A})$, and $\left(B^{*}\right)^{\mathfrak{M}, i}=\{r \in \mathfrak{R} \mid B \in r(i)\}$, for each $B \in \operatorname{cl}(\mathcal{K})$ and $i \geq 0$. It should be clear that $(\mathfrak{M}, 0) \models \mathcal{K}^{\ddagger}$.

Theorem 2. If there is a quasimodel $\mathfrak{Q}=\left\langle W, K, K_{0}, L\right\rangle$ for $\mathcal{K}$ then there is an ultimately periodic quasimodel $\mathfrak{Q}^{\prime}=\left\langle W^{\prime}, K, K_{0}, L^{\prime}\right\rangle$ for $\mathcal{K}$, that is, there are $L^{\prime} \leq L+2^{N}$ and $P \leq N \cdot 2^{N}$, where $N=|W| \cdot|c l(\mathcal{K})|$, such that $r^{\prime}(i+P)=r^{\prime}(i)$, for all $i \geq L^{\prime}$ and $\left(r^{\prime}, \Xi^{\prime}\right) \in W^{\prime}$.

Proof. The proof is a straightforward modification of the standard $\mathcal{L} \mathcal{T}$ construction (see, e.g., [14]) with the set of propositions being $\operatorname{cl}(\mathcal{K}) \times W$.

It follows from Lemma 2 and Theorems 1 and 2 that we have the following:

Theorem 3. Satisfiability of $T_{\mathcal{U}} D L-L i t e_{\text {bool }}^{(\mathcal{R N})} K B$ s is PSPACE-complete. 


\section{Satisfiability of $T_{\diamond} D L$-Lite bool $_{(\mathcal{R N})}$ KBs is NP-complete}

We notice that Lemma 2 holds for $T_{\diamond} D L-L i t e_{b o o l}^{(\mathcal{R N})}$ and its respective fragment $T_{\diamond}^{0} D L-L i t e_{b o o l}^{\mathcal{N}}$. Thus, to prove that satisfiability of $T_{\diamond} D L-L i t e_{\text {bool }}^{(\mathcal{R N})} \mathrm{KBs}$ is in NP, it is enough to consider $T_{\diamond}^{0} D L-L i t e_{\text {bool }}^{\mathcal{N}}$ KBs. We proceed as in Section 4 . First we prove that a $T_{\diamond}^{0} D L-L i t e_{b o o l}^{\mathcal{N}} \mathrm{KB}$ is satisfiable iff there exists a quasimodel for it. Then we show that if there is a quasimodel for $\mathcal{K}$ then there exists an ultimately periodic quasimodel for $\mathcal{K}$ such that both the length of the prefix and the length of the period are polynomial in the length of $\mathcal{K}$. As the existence of such a quasimodel can be checked in non-deterministic polynomial time, we obtain the NP upper bound. The matching lower bound will be shown for a sublogic $T_{\diamond} D L$-Lite core $_{\text {of }} T_{\diamond} D L$-Lite bool $_{(\mathcal{R N})}$ with rather primitive concept inclusions.

Let $\mathcal{K}=(\mathcal{T}, \mathcal{A})$ be a $T_{\diamond}^{0} D L-L i t e_{\text {bool }}^{\mathcal{N}} \mathrm{KB}$. We say that a type $\mathbf{t}$ for $\mathcal{K}$ is stutterinvariant if $\neg \diamond C \in \mathbf{t}$ implies $\neg C \in \mathbf{t}$, for each $\diamond C \in e v(\mathcal{K})$. A quasimodel for $\mathcal{K}$ is a triple $\mathfrak{Q}=\langle W, K, L\rangle$, where $W$ is a set of witnesses for $\mathcal{K}$ and $K, L$ are natural numbers with $0 \leq K \leq L$ such that they satisfy (run), (obj), (role) and the following condition

(stuttr) $r(K)$ and the $r(i)$, for $i \geq L$, are stutter-invariant for each $(r, \Xi) \in W$.

Theorem 4. $A T_{\diamond}^{0} D L-$ Lit $_{\text {bool }}^{\mathcal{N}} K B \mathcal{K}$ is satisfiable iff there is a quasimodel $\mathfrak{Q}=$ $\langle W, K, L\rangle$ for $\mathcal{K}$ such that $L \leq N_{\mathcal{A}}+|e v(\mathcal{K})| \cdot\left(\mid\right.$ role $\left.^{ \pm}(\mathcal{K}) \mid+2\right)+3$.

Proof. $(\Rightarrow)$ Suppose $\mathcal{I} \models \mathcal{K}$. Let $V=|e v(\mathcal{K})|$. By Lemma 3, there exists $M$ with $N_{\mathcal{A}} \leq M \leq N_{\mathcal{A}}+V \cdot\left|\mathbf{F}^{0}\right|$ such that, for every role $R \in \mathbf{F}^{0}$, either $R \in \mathbf{F}^{K}$ or $R \notin \mathbf{F}^{M+1}$, where $K=M+V+1$. We then set

$$
i_{R}= \begin{cases}\min \left\{i \geq K \mid R^{\mathcal{I}(i)} \neq \emptyset\right\}, & R \in \mathbf{F}^{K}, \\ \max \left\{i \mid R^{\mathcal{I}(i)} \neq \emptyset\right\}, & R \in \mathbf{F}^{0} \backslash \mathbf{F}^{M+1} .\end{cases}
$$

Clearly, for $R \in \mathbf{F}^{0}$, either $i_{R} \leq M$ or $i_{R} \geq K$. For each $R \in \mathbf{F}^{0}$, we fix some $d_{R} \in(\exists R)^{\mathcal{I}\left(i_{R}\right)}$ and set $r_{R}=\operatorname{run}_{\mathcal{I}}\left(d_{R}\right)$. For $a \in o b(\mathcal{A})$, set $r_{a}=\operatorname{run}_{\mathcal{I}}\left(a^{\mathcal{I}}\right)$. Let

$$
W=\left\{\left(r_{a}, \emptyset\right) \mid a \in o b(\mathcal{A})\right\} \cup\left\{\left(r_{R},\left\{i_{R}\right\}\right) \mid R \in \mathbf{F}^{0}\right\} .
$$

Clearly, (run) and (obj) hold. Also, we have $\exists R^{-} \in r(i)$ iff $\exists R \in r_{R}\left(i_{R}\right)$ and $\left(r_{R},\left\{i_{R}\right\}\right) \in W$, for all $(r, \Xi) \in W$ and $i \geq 0$.

We now transform $W$ by expanding and pruning runs in such a way that the $r(i)$ are never thrown out for $(r, \Xi) \in W$ and $i \in \Xi$.

Lemma 4. For each coherent and saturated run $r$,

$$
\mid\{i \mid r(i) \text { is not stutter-invariant }\}|\leq| e v(\mathcal{K}) \mid .
$$

Proof. Suppose that there are $0 \leq i_{1}<\cdots<i_{n}$ such that $n>|\operatorname{ev}(\mathcal{K})|$ and $r\left(i_{1}\right), \ldots, r\left(i_{n}\right)$ are not stutter-invariant, i.e., there are $\diamond C_{j} \in \operatorname{ev}(\mathcal{K})$ with $\neg \diamond C_{j}, C_{j} \in r\left(i_{j}\right)$. Then there is $\diamond C \in \operatorname{ev}(\mathcal{K})$ such that $\neg \diamond C, C \in r\left(i_{j}\right), r\left(i_{j^{\prime}}\right)$ 
for some $0 \leq i_{j}<i_{j^{\prime}}$. As $C \in r\left(i_{j^{\prime}}\right)$, we have, by (coh), $\diamond C \in r\left(i_{j}\right)$, contrary to $\neg \diamond C \in r\left(i_{j}\right)$.

Step 1. By Lemma 4, for each $(r, \Xi) \in W$, there is $j_{r}, M<j_{r} \leq K$, such that $r\left(j_{r}\right)$ is stutter-invariant. Set

$$
\begin{aligned}
r^{\prime} & =r^{<j_{r}} \cdot r\left(j_{r}\right)^{K-j_{r}} \cdot r^{\geq j_{r}}, \\
\Xi^{\prime} & =\left\{i \mid i \in \Xi, i \leq j_{r}\right\} \cup\left\{i+K-j_{r} \mid i \in \Xi, i>j_{r}\right\} .
\end{aligned}
$$

Clearly, $r^{\prime}$ is a coherent and saturated run. Denote by $W^{\prime}$ the set of all $\left(r^{\prime}, \Xi^{\prime}\right)$ constructed as above. Then, for each $\left(r^{\prime}, \Xi^{\prime}\right) \in W^{\prime}, r^{\prime}(K)$ is stutter-invariant. It is easy to see that, for each $R \in \mathbf{F}^{0},\left(r_{R}^{\prime},\left\{i_{R}^{\prime}\right\}\right) \in W^{\prime}$ and either $i_{R}^{\prime} \leq M$ or $i_{R}^{\prime} \geq K$.

Step 2. For $\left(r^{\prime}, \Xi^{\prime}\right) \in W^{\prime}$, let $\Xi^{0}=\left\{i>K \mid r^{\prime}(i)\right.$ is not stutter-invariant $\}$. By Lemma $4,\left|\Xi^{0}\right| \leq|e v(\mathcal{K})|$. If $\Xi^{0} \cup \Xi^{\prime} \neq \emptyset$, we prune the run $r^{\prime}$ by removing all stutter-invariant $r^{\prime}(i)$ with $K<i<\max \left(\Xi^{0} \cup \Xi^{\prime}\right)$. The resulting function $r^{\prime \prime}$ is a coherent and saturated run for $\mathcal{K}$. Set

$$
\Xi^{\prime \prime}=\left\{i \mid i \in \Xi^{\prime}, i \leq K\right\} \cup\left\{K+\sharp\left\{j \in \Xi^{0} \cup \Xi^{\prime} \mid j \leq i\right\} \mid i \in \Xi^{\prime}, i>K\right\} .
$$

Let $W^{\prime \prime}$ be the set of all witnesses $\left(r^{\prime \prime}, \Xi^{\prime \prime}\right)$ constructed as above and $L=K+$ $V+2$. Clearly, for each $\left(r^{\prime \prime}, \Xi^{\prime \prime}\right) \in W^{\prime \prime}$, all the types $r^{\prime \prime}(i)$ are stutter-invariant, for $i \geq L$. Thus, (stuttr) holds. It is easy to see that, for each $R \in \mathbf{F}^{0}$, we have $\left(r_{R}^{\prime \prime},\left\{i_{R}^{\prime \prime}\right\}\right) \in W^{\prime \prime}$ and $K \leq i_{R}^{\prime \prime}<L$ if $R \in \mathbf{F}^{K}$, and $i_{R}^{\prime \prime} \leq M$ if $R \notin \mathbf{F}^{M+1}$. So (role) holds as well. It is readily seen now that $\mathfrak{Q}=\left\langle W^{\prime \prime}, K, L\right\rangle$ is as required.

$(\Leftarrow)$ Let $\mathfrak{Q}=\langle W, K, L\rangle$ be a quasimodel for $\mathcal{K}$. We construct a model for $\mathcal{K}^{\ddagger}$ which, by Theorem 1 , will show that $\mathcal{K}$ is satisfiable. Let

$$
\begin{array}{r}
\mathfrak{R}=\left\{r_{a} \mid\left(r_{a}, \emptyset\right) \in W\right\} \cup\left\{r_{\bar{R}}^{\geq i} \mid\left(r_{R},\left\{i_{R}\right\}\right) \in W, 0 \leq i \leq i_{R}\right\} \cup \\
\left\{r_{R}^{<K} \cdot\left(r_{R}(K)\right)^{i-i_{R}} \cdot r_{R}^{\geq K} \mid\left(r_{R},\left\{i_{R}\right\}\right) \in W, i>i_{R} \geq K\right\} .
\end{array}
$$

Clearly, each $r \in \mathfrak{R}$ is a coherent and saturated run for $\mathcal{K}$. Moreover, if we have $\left(r_{R},\left\{i_{R}\right\}\right) \in W$ and $i_{R}<K$ then, for all $i, 0 \leq i \leq i_{R}$, there is $r^{\prime} \in \mathfrak{R}$ with $\exists R \in r^{\prime}(i)$. And if $\left(r_{R},\left\{i_{R}\right\}\right) \in W$ and $i_{R} \geq K$ then, for all $i \geq 0$, there is $r^{\prime} \in \mathfrak{R}$ with $\exists R \in r^{\prime}(i)$. As follows from (role), for each $R \in \Omega$, we have $R^{-} \in \Omega$ and either $i_{R} \geq K$ and $i_{R^{-}} \geq K$ or $i_{R}=i_{R^{-}}<K$. So, for all $i \geq 0$ and $r \in \mathfrak{R}$,

$$
\text { if } \exists R^{-} \in r(i) \text { then there is } r^{\prime} \in \mathfrak{R} \text { such that } \exists R \in r^{\prime}(i) \text {. }
$$

We construct a first-order temporal model $\mathfrak{M}$ based on the domain $D=\mathfrak{R}$ by taking $a^{\mathfrak{M}}=r_{a}$, for each $a \in o b(\mathcal{A})$, and $\left(B^{*}\right)^{\mathfrak{M}, i}=\{r \in \mathfrak{R} \mid B \in r(i)\}$, for each $B \in \operatorname{cl}(\mathcal{K})$ and $i \geq 0$. It should be clear that $(\mathfrak{M}, 0) \models \mathcal{K}^{\ddagger}$.

Theorem 5. If there is a quasimodel $\mathfrak{Q}=\langle W, K, L\rangle$ for $\mathcal{K}$ then there is an ultimately periodic quasimodel $\mathfrak{Q}^{\prime}=\left\langle W^{\prime}, K, L\right\rangle$, that is, there is $P \leq|\operatorname{ev}(\mathcal{K})|$ such that $r^{\prime}(i+P)=r^{\prime}(i)$, for all $i>L$ and $\left(r^{\prime}, \Xi^{\prime}\right) \in W^{\prime}$. 
Proof. We begin the proof with the following observation:

Lemma 5. Let $r$ be a coherent and saturated run for $\mathcal{K}$ and let $l \geq 0$ be such that every $r(i), i \geq l$, is stutter-invariant. Then there are $i_{1}, \ldots, i_{|e v(\mathcal{K})|} \geq l$ such that $r^{\prime}=r \leq l \cdot\left(r\left(i_{1}\right) \cdot \ldots \cdot r\left(i_{|e v(\mathcal{K})|}\right)\right)^{\omega}$ is a coherent and saturated run for $\mathcal{K}$.

Proof. First we show that

$$
r(l) \cap e v(\mathcal{K})=r(j) \cap e v(\mathcal{K}), \quad \text { for all } j>l .
$$

Suppose that there is $j>l$ and $\diamond C \in r(l)$ such that $\diamond C \notin r(j)$. As $r(j)$ is stutterinvariant, $C \notin r(j)$ and, by (coh), $\diamond C \notin r(j-1)$. By repeating this argument sufficiently many times, we obtain $\diamond C \notin r(l)$, contrary to our assumption. The converse direction-i.e., for each $j>l$, if $\diamond C \in r(j)$ then $\diamond C \in r(l)$-follows from (coh).

For each $\diamond C \in e v(\mathcal{K})$, we can select an $i, i \geq l$, such that $C \in r(i)$ whenever $\diamond C \in r(l)$. Let $i_{1}, \ldots, i_{|e v(\mathcal{K})|}$ be all such $i$. It remains to show that $r^{\prime}$ is coherent and saturated. For coherency of $r^{\prime}$, let $C \in r^{\prime}(i)$, for $i \geq 0$. By (coh) for $r$, we have $\diamond C \in r^{\prime}(j)$, for each $0 \leq j<i$ such that $j \leq l$. It remains to consider $j$ with $l<j<i$. It follows that $r^{\prime}(i)=r\left(i_{k}\right)$, for some $1 \leq k \leq|e v(\mathcal{K})|$, from which, by (coh) for $r, \diamond C \in r(l)=r^{\prime}(l)$ and, by $(5), \diamond C \in r^{\prime}(j)$. For saturation of $r^{\prime}$, let $\diamond C \in r^{\prime}(i)$, for $i \geq 0$. If $\diamond C \in r(l)$ then $C \in r\left(i_{k}\right)$ for $1 \leq k \leq|e v(\mathcal{K})|$ and, by the construction of $r^{\prime}$, there is $j>i$ such that $r^{\prime}(j)=r\left(i_{k}\right)$. Thus $C \in r^{\prime}(j)$. If $\diamond C \notin r(l)$ then, by (5), $i<l$, from which $\diamond C \in r(i)$. By (sat) for $r$, there is $j>i$ with $C \in r(j)$ and, by $(5), j \leq l$. Thus $C \in r(j)=r^{\prime}(j)$.

Let $P=|e v(\mathcal{K})|$. For each $(r, \Xi)$, we take $r^{\prime}=r^{\leq L} \cdot\left(r\left(i_{1}\right) \cdot \ldots r\left(i_{P}\right)\right)^{\omega}$ provided by Lemma 5 . Denote the set of all $\left(r^{\prime}, \Xi\right)$ by $W^{\prime}$. It follows that $\mathfrak{Q}^{\prime}=\left\langle W^{\prime}, K, L\right\rangle$ is an ultimately periodic quasimodel for $\mathcal{K}$ (with period $P$ ).

It is now easy to devise an NP algorithm which can check whether there exists a quasimodel for a $T_{\diamond}^{0} D L-L i t e_{\text {bool }}^{\mathcal{N}}$ KB. By Lemma 2 and Theorems 4 and 5 , this means that satisfiability of $T_{\diamond} D L-L i t e_{\text {bool }}^{(\mathcal{R N})} \mathrm{KBs}$ is in NP. We prove the matching lower bound for the fragment $T_{\diamond} D L-$ Lite $_{\text {core }}$ of $T_{\diamond} D L-L i t e_{\text {bool }}^{(\mathcal{R N})}$ that allows only concept inclusions of the form $A_{1} \sqsubseteq A_{2}, A_{1} \sqsubseteq \neg A_{2}, \diamond A_{1} \sqsubseteq A_{2}$ or $A_{1} \sqsubseteq \diamond A_{2}$, where $A_{1}$ and $A_{2}$ are concept names.

Lemma 6. The satisfiability problem for $T_{\diamond} D L-L i t e_{\text {core }} K B s$ is NP-hard.

Proof. We prove this by reduction of the graph 3-colourability (3-CoL) problem, which is formulated as follows: given a graph $G=(V, E)$, decide whether there is an assignment of colours $\{1,2,3\}$ to vertices $V$ such that no two vertices $a_{i}, a_{j} \in V$ sharing the same edge, $\left(a_{i}, a_{j}\right) \in E$, have the same colour. Let $X_{0}$, $X_{1}, X_{2}, V, U$ and $A_{i}$, for $A_{i} \in V$, be concept names and $a$ an object name. Consider the following $\mathrm{KB} \mathcal{K}_{G}$ :

$$
\begin{array}{ll}
V(a), \quad V \sqsubseteq \diamond A_{i}, \quad A_{i} \sqsubseteq X_{3}, \quad \text { for all } A_{i} \in V, \\
A_{i} \sqsubseteq \neg A_{j}, \quad \text { for all }\left(A_{i}, A_{j}\right) \in E, \\
V \sqsubseteq \neg U, \quad \diamond X_{0} \sqsubseteq U, \quad \diamond X_{1} \sqsubseteq X_{0}, \quad \diamond X_{2} \sqsubseteq X_{1}, \quad \diamond X_{3} \sqsubseteq X_{2} .
\end{array}
$$


It is easy to see that $\mathcal{K}_{G}$ is satisfiable iff $G$ is 3 -colourable.

Thus we obtain the following theorem:

Theorem 6. The satisfiability problem for $T_{\diamond D L-L i t e_{\text {bool }}^{(\mathcal{R N})}}$ KBs is NP-complete.

It is also of interest to note that the fragment $T_{\mathcal{U}} D L-L i t e_{\text {core }}$ of $T_{\mathcal{U}}^{0} D L-L i t e_{\text {bool }}^{\mathcal{N}}$ with concept inclusions of the form $A_{1} \sqsubseteq A_{2}, A_{1} \sqsubseteq \neg A_{2}$ or $A_{1} \sqsubseteq A_{2} \mathcal{U} A_{3}$ (the $A_{i}$ concept names) turns out to be as complex as the whole logic $T_{\mathcal{U}} D L-L i t e_{\text {bool }}^{(\mathcal{R N})}$ :

Theorem 7. The satisfiability problem for $T_{\mathcal{U}} D L-L i t$ Lore $_{\text {co }}$ KB is PSPACE-hard.

The proof can be found in the full version of the paper available online at http://www.dcs.bbk.ac.uk/ roman/.

\section{Conclusions}

The obtained complexity results look encouraging in view of possible applications for reasoning about temporal conceptual data models [4]. On the one hand, the logic $D L-L i t e_{\text {bool }}^{\mathcal{N}}$ was shown to be adequate for representing different aspects of conceptual models: ISA, disjointness and covering for classes, domain and range of relationships, $n$-ary relationships, attributes and participation constraints [6]. On the other hand, the approach of [8] shows that rigid axioms and roles with temporalised concepts are enough to capture temporal data models.

The logic $T_{\diamond} D L-L_{i t e}$ bool presented in this paper can capture some form of evolution constraints $[5,20,16]$ thanks to the $\diamond$ operator. Furthermore, it also captures snapshot classes - i.e., classes whose instances do not change over time. However, by restricting the temporal component only to $\diamond$ and $\square$, we lose the ability to capture temporary entities and relationships whose instances have a limited lifespan. To overcome this limitation, we plan to extend the logics presented here with either past temporal operators or with a special kind of axioms that hold over finite prefix.

\section{References}

1. A. Artale, D. Calvanese, R. Kontchakov, and M. Zakharyaschev. DL-Lite in the light of first-order logic. In Proc. of the 22nd AAAI Conf. on Artificial Intelligence, pages 361-366. AAAI Press, 2007.

2. A. Artale, D. Calvanese, R. Kontchakov, and M. Zakharyaschev. The DL-Lite family and relations. Technical Report BBKCS-09-03, School of Computer Science and Information Systems, Birbeck College, London, 2009. Available at http:// www.dcs.bbk.ac.uk/research/techreps/2009/bbkcs-09-03.pdf.

3. A. Artale and E. Franconi. Temporal description logics. In M. Fisher, D. Gabbay, and L. Vila, editors, Handbook of Time and Temporal Reasoning in Artificial Intelligence, pages 375-388. Elsevier, 2005. 
4. A. Artale, E. Franconi, F. Wolter, and M. Zakharyaschev. A temporal description logic for reasoning about conceptual schemas and queries. In Proc. of the 8th Joint European Conf. on Logics in Artificial Intelligence (JELIA), volume 2424 of LNAI, pages 98-110. Springer, 2002.

5. A. Artale, C. Parent, and S. Spaccapietra. Evolving objects in temporal information systems. Annals of Mathematics and AI, 50(1-2):5-38, 2007.

6. A. Artale, D. Calvanese, R. Kontchakov, V. Ryzhikov, and M. Zakharyaschev. Reasoning over extended ER models. In Proc. of the 26th Int. Conf. on Conceptual Modeling (ER), pages 277-292, volume 4801 of LNCS. Springer, 2007.

7. A. Artale, R. Kontchakov, C. Lutz, F. Wolter, and M. Zakharyaschev. Temporalising tractable description logics. In Proc. of the 14th Int. Symposium on Temporal Representation and Reasoning (TIME). IEEE Computer Society, 2007.

8. A. Artale, C. Lutz, and D. Toman. A description logic of change. In Proc. of Int. Joint Conf. on Artificial Intelligence (IJCAI), pages 218-223, 2007.

9. F. Baader, R. Küsters, and F. Wolter. Extensions to description logics. In Description Logic Handbook, pages 219-261. Cambridge University Press, 2003.

10. F. Baader, S. Ghilardi, and C. Lutz. LTL over description logic axioms. In Proc. of the 11th Int. Conf. on Principles of Knowledge Representation and Reasoning (KR), pages 684-694. AAAI Press, 2008.

11. D. Calvanese, G. De Giacomo, D. Lembo, M. Lenzerini, and R. Rosati. DL-Lite: Tractable description logics for ontologies. In Proc. of the 10th Nat. Conf. on Artificial Intelligence and the 17th Innovative Applications of Artificial Intelligence Conf., pages 602-607, AAAI Press/The MIT Press, 2005.

12. D. Calvanese, G. De Giacomo, D. Lembo, M. Lenzerini, and R. Rosati. Data complexity of query answering in description logics. In Proc. of the 10th Int. Conf. on Principles of Knowledge Representation and Reasoning (KR), pages 260-270. AAAI Press, 2006.

13. D. Calvanese, G. De Giacomo, D. Lembo, M. Lenzerini, and R. Rosati. Tractable reasoning and efficient query answering in description logics: The DL-Lite family. J. of Automated Reasoning, 39(3):385-429, 2007.

14. D. Gabbay, I. Hodkinson and M. Reynolds. Temporal Logic: Mathematical Foundations and Computational Aspects, Volume 1. Oxford University Press, 1994.

15. D. Gabbay, A. Kurucz, F. Wolter, and M. Zakharyaschev. Many-Dimensional Modal Logics: Theory and Applications. Elsevier, 2003.

16. G. Hall and R. Gupta. Modeling transition. In Proc. of the 7th Int. Conf. on Data Engineering (ICDE), pages 540-549. IEEE Computer Society, 1991.

17. C. Lutz, F. Wolter, and M. Zakharyaschev. Temporal description logics: A survey. In Proc. of the 14th Int. Symposium on Temporal Representation and Reasoning (TIME), pages 3-14. IEEE Computer Society, 2008.

18. A. Poggi, D. Lembo, D. Calvanese, G. De Giacomo, M. Lenzerini, and R. Rosati. Linking data to ontologies. J. on Data Semantics, X:133-173, 2008.

19. K. Schild. Combining terminological logics with tense logic. In Proc. of the 6th Portuguese Conf. on Artificial Intelligence (EPIA), volume 727 of LNCS, pages 105-120. Springer, 1993.

20. S. Spaccapietra, C. Parent, and E. Zimanyi. Conceptual Modeling for Traditional and Spatio-Temporal Applications-The MADS Approach. Springer, 2006. 


\section{A Proof of Theorem 7}

Theorem 7. The satisfiability problem for $T_{\mathcal{U}} D L-L i t e_{\text {core }}$ KBs is PSPACE-hard.

Proof. We show that the unsatisfiability problem for $T_{\mathcal{U}} D L-L i t e_{\text {core }} \mathrm{KBs}$ is PSPACE-hard. The proof is by reduction of the halting problem of deterministic Turing machines that require polynomial space $s(n)$ given an input of length $n$. Without loss of generality, assume $s(n)$ is computable in time polynomial of $n$ and that the Turing machine $M$ halts on all inputs (either in accepting or rejecting state) and never runs outside the first $s(n)$ tape cells (we also assume it never goes to the left of the input).

Let $M=\left\langle Q, \Sigma, \Gamma, \delta, q_{0}, \#, q_{a}, q_{r}\right\rangle$, where $Q$ is a finite set of states, $\Gamma$ is a set of tape symbols, \# $\# \Gamma$ is the blank symbol, $\Sigma \subseteq \Gamma$ is a set of input symbols, $\delta:\left(Q \backslash\left\{q_{r}, q_{a}\right\}\right) \times \Gamma \rightarrow Q \times \Gamma \times\{L, R\}$ is a transition function, $q_{0}, q_{r}, q_{a} \in Q$ are the initial, accepting and rejecting states, respectively.

Let $\boldsymbol{a}=a_{1} \ldots a_{n}$ be an input for $M$. We define a KB $\tau(M, \boldsymbol{a})$ that is unsatisfiable iff $M$ accepts $\boldsymbol{a}$. We use the following concept names, for $1 \leq i \leq s(n)$,

- $H_{i} q, q \in Q$, with the intended meaning that $H_{i} q$ is true iff the head points to the cell $i$ and the current control state is $q$;

- $S_{i} a, a \in \Gamma$, with the intended meaning that $S_{i} a$ is true iff the tape cell $i$ contains symbol $a$;

- $D_{i}$ with the intended meaning that $D_{j}$ is false, for all $j \neq i$, if in the previous configuration the head pointed to the cell $i$

- $P_{i} q, q \in Q$, with the intended meaning that $P_{i} q$ is true if in the previous configuration the head pointed to the cell $i$ and the state was $q ; \overline{P_{i} q}$ has the opposite meaning.

Consider the TBox $\mathcal{T}$ containing the following concept inclusions, for all $a, a^{\prime} \in \Gamma, q \in Q \backslash\left\{q_{r}, q_{a}\right\}, q^{\prime} \in Q$ and $1 \leq i \leq s(n)$,

$$
\begin{array}{rlrl}
H_{i} q & \sqsubseteq \mathcal{U} P_{i} q, & & \\
P_{i} q & \sqsubseteq \neg \overline{P_{i} q}, & & \\
P_{i} q \sqsubseteq \neg D_{j} & & \text { for all } 1 \leq j \leq s(n), \quad j \neq i, \\
S_{i} a & \sqsubseteq S_{i} a \mathcal{U} D_{i}, & & \\
S_{i} a & \sqsubseteq S_{i} a^{\prime} \mathcal{U} \overline{P_{i} q}, & & \text { if } \delta(q, a)=\left(q^{\prime}, a^{\prime}, R\right) \text { or } \delta(q, a)=\left(q^{\prime}, a^{\prime}, L\right), \\
S_{i} a & \sqsubseteq H_{i+1} q^{\prime} \mathcal{U} \overline{P_{i} q}, & & \text { if } \delta(q, a)=\left(q^{\prime}, a^{\prime}, R\right) \text { and } i<s(n), \\
S_{i} a & \sqsubseteq H_{i-1} q^{\prime} \mathcal{U} \overline{P_{i} q}, & & \text { if } \delta(q, a)=\left(q^{\prime}, a^{\prime}, L\right) \text { and } i>1,
\end{array}
$$

and the following $\mathrm{ABox} \mathcal{A}$ :

$$
H_{1} q_{0}(d), \quad S_{i} a_{i}(d), 1 \leq i \leq n, \quad S_{i} \#(d), n<i \leq s(n), \quad \square R(d) .
$$

Note that although $\perp$ is not available in $T_{\mathcal{U}} D L-L i t e_{\text {core }}$, it can be easily introduced as a concept name $B$ with the following concept inclusions: $B \sqsubseteq A$ and $B \sqsubseteq \neg A$, for some fresh concept name $A$. Let

$$
\tau(M, \boldsymbol{a})=\left(\mathcal{T} \cup\left\{R \sqsubseteq \neg H_{i} q_{a} \mid 1 \leq i \leq s(n)\right\}, \mathcal{A}\right) .
$$


We are going to show that $\tau(M, \boldsymbol{a})$ is unsatisfiable iff $M$ accepts $\boldsymbol{a}$. We represent configurations of $M$ as tuples of the form $\mathfrak{c}=\left\langle b_{1} \ldots b_{s(n)}, i, q\right\rangle$, where $b_{1} \ldots b_{s(n)}$ is the contents of the first $s(n)$ tape cells $\left(b_{j} \in \Gamma\right.$, for $\left.1 \leq j \leq s(n)\right), i$ is the head position, $1 \leq i \leq s(n)$, and $q$ is the control state, $q \in Q$. We say that a point $d \in \Delta^{\mathcal{I}}$ encodes a configuration $\mathfrak{c}=\left\langle b_{1} \ldots b_{s(n)}, i, q\right\rangle$ at moment $k$ in $\mathcal{I}$ if $d \in\left(H_{i} q\right)^{\mathcal{I}(k)}$ and $d \in\left(S_{j} b_{j}\right)^{\mathcal{I}(k)}$, for all $1 \leq j \leq s(n)$.

Claim. Let $\mathfrak{c}_{0}, \ldots, \mathfrak{c}_{m}$ be a sequence of configurations representing a partial computation of $M$ on $\boldsymbol{a}$. Then for any model $\mathcal{I}$ of $(\mathcal{T}, \mathcal{A}), d^{\mathcal{I}}$ encodes $\mathfrak{c}_{k}$ at moment $k$ in $\mathcal{I}$, for all $0 \leq k \leq m$.

Proof. The proof is by induction on $k$. For $k=0$ the claim follows from $\mathcal{I} \models \mathcal{A}$. For the induction step, let $d^{\mathcal{I}}$ encode $\mathfrak{c}_{k}=\left\langle b_{1} \ldots b_{i} \ldots b_{s(n)}, i, q\right\rangle$ at moment $k$ in $\mathcal{I}$ and let $\mathfrak{c}_{k+1}=\left\langle b_{1} \ldots b_{i}^{\prime} \ldots b_{s(n)}, i^{\prime}, q^{\prime}\right\rangle$. Consider first $\delta\left(q, b_{i}\right)=\left(q^{\prime}, b_{i}^{\prime}, L\right)$, in which case $i>1$ and $i^{\prime}=i-1$. Then $d^{\mathcal{I}} \in H_{i} \mathcal{I}^{\mathcal{I}(k)}$ for $q \notin\left\{q_{r}, q_{a}\right\}$ and, by (6), $d^{\mathcal{I}} \in P_{i} q^{\mathcal{I}(k+1)}$. By $(7), d^{\mathcal{I}} \notin{\overline{P_{i} q}}^{\mathcal{I}(k+1)}$, and, as $d^{\mathcal{I}} \in S_{i} b_{i}^{\mathcal{I}(k)}$, we obtain, by (12), $d^{\mathcal{I}} \in H_{i^{\prime}} q^{\mathcal{I}(k+1)}$ and, by $(10), d^{\mathcal{I}} \in S_{i} b_{i}^{\mathcal{I}(k+1)}$. Let $b_{j}$ for $j \neq i, 1 \leq j \leq s(n)$. By (8), $d^{\mathcal{I}} \notin D_{j}^{\mathcal{I}(k+1)}$, and so, since $d^{\mathcal{I}} \in S_{j} b_{j}^{\mathcal{I}(k)}$, we get, by $(9), d^{\mathcal{I}} \in S_{j} b_{j}^{\mathcal{I}(k+1)}$. Hence, $d^{\mathcal{I}}$ encodes $\mathfrak{c}_{k+1}$ at moment $k+1$ in $\mathcal{I}$. The case of $\delta\left(q, b_{i}\right)=\left(q^{\prime}, b_{i}^{\prime}, R\right)$ is similar.

It follows that if $M$ accepts $\boldsymbol{a}$ then $\tau(M, \boldsymbol{a})$ is unsatisfiable. Indeed, if $M$ accepts $\boldsymbol{a}$ then the computation is a sequence of configurations $\mathfrak{c}_{0}, \ldots, \mathfrak{c}_{k}$ such that $\mathfrak{c}_{k}=\left\langle b_{1} \ldots b_{i} \ldots b_{s(n)}, i, q_{a}\right\rangle$. Suppose $\tau(M, \boldsymbol{a})$ is satisfiable, i.e., there is a model $\mathcal{I}$ of $\tau(M, \boldsymbol{a})$, which is also a model of $(\mathcal{T}, \mathcal{A})$. By the above claim, we have $d^{\mathcal{I}} \in H_{i} q_{a}^{\mathcal{I}(k)}$, which implies $d^{\mathcal{I}} \notin R^{\mathcal{I}(k)}$ contrary to our assumption.

Conversely, if $M$ rejects $\boldsymbol{a}$ then $\tau(M, \boldsymbol{a})$ is satisfiable. Let $\mathfrak{c}_{0}, \ldots, \mathfrak{c}_{m}$ be a sequence of configurations representing the rejecting computation of $M$ on $\boldsymbol{a}$, $\mathfrak{c}_{k}=\left\langle b_{1, k}, \ldots, b_{s(n), k}, i_{k}, q_{k}\right\rangle$, for $0 \leq k \leq m$. We define an interpretation $\mathcal{I}$ with domain $\Delta^{\mathcal{I}}=\{w\}$. Let $d^{\mathcal{I}}=w$. For every $0 \leq k \leq m, 1 \leq j \leq s(n), a \in \Gamma$, $q \in Q$, we set

$-R^{\mathcal{I}(k)}=\Delta^{\mathcal{I}}$,

- $H_{j} q_{\mathcal{I}(k)}^{\mathcal{I}(k)} \dot{\Delta}^{\mathcal{I}}$ if $j=i_{k}$ and $q=q_{k}, \emptyset$ otherwise,

$-S_{j} a^{\mathcal{I}(k)}=\Delta^{\mathcal{I}}$ if $a=b_{j, k}$ and $\emptyset$ otherwise,

- $P_{j} q^{\mathcal{I}(0)}=\emptyset$ and $P_{j} q^{\mathcal{I}(k+1)}=H_{j} q^{\mathcal{I}(k)}$ if $k+1 \leq m, \quad{\overline{P_{j}}}^{\mathcal{I}(k)}=\Delta^{\mathcal{I}} \backslash P_{j} q^{\mathcal{I}(k)}$,

- $D_{j}^{\mathcal{I}(k)}=\bigcup_{q^{\prime} \in Q} P_{j} q^{\prime \mathcal{I}(k)}$,

For every $k>m, 1 \leq j \leq s(n), a \in \Gamma, q \in Q$, we set

- $R^{\mathcal{I}(k)}=\Delta^{\mathcal{I}}$,

- $H_{j} q^{\mathcal{I}(k)}=\Delta^{\mathcal{I}}$ if $q=q_{r}$ and $\emptyset$ otherwise,

$-S_{j} a^{\mathcal{I}(k)}=\emptyset$,

- $P_{j} q^{\mathcal{I}(k)}=\emptyset$ and ${\overline{P_{j}}}^{\mathcal{I}(k)}=\Delta^{\mathcal{I}}$,

$-D_{j}^{\mathcal{I}(k)}=\Delta^{\mathcal{I}}$.

It can be easily verified that $\mathcal{I} \models \tau(M, \boldsymbol{a})$. 


\section{B Decision procedure for $T_{\mathcal{U}}^{0} D L-L i t e_{b o o l}^{\mathcal{N}}$}

First we guess and store in binary numbers $K, K_{0}, L^{\prime}$ and $P$ such that

$$
\begin{aligned}
& 0<K<K_{0}<L^{\prime} \leq N_{\mathcal{A}}+T^{\left|\operatorname{role}^{ \pm}(\mathcal{K})\right|} \cdot\left(\left|\operatorname{role}^{ \pm}(\mathcal{K})\right|+1\right)+T+2+2^{N}, \\
& 0<P \leq N \cdot 2^{N}
\end{aligned}
$$

where $N \leq\left(\left|\operatorname{role}^{ \pm}(\mathcal{K})\right|+|o b(\mathcal{A})|\right) \cdot|\operatorname{cl}(\mathcal{K})|$. Then we guess a set $\Omega \subseteq$ role $^{ \pm}(\mathcal{K})$ and numbers $\left\{i_{R} \mid R \in \Omega\right\}$ such that $i_{R}<L^{\prime}$, for each $R \in \Omega$. Let $\Delta=\Omega \cup o b(\mathcal{A})$.

Let $\left\{r_{x}(i) \mid x \in \Delta\right\}$ be a state of an ultimately periodic quasimodel (cf. Theorem 2). Consider the following conditions:

1. $B \in r_{a}(i)$, for all $\bigcirc^{i} B(a) \in \mathcal{A} ; B \in r_{a}(i)$, for all $\square B(a) \in \mathcal{A}$;

2. $r_{x}(i)$ is realisable, for all $x \in \Omega \cup o b(\mathcal{A})$;

3. for all $R \in \operatorname{role}^{ \pm}(\mathcal{K})$, if $i_{R}=i$ then $\exists R \in r_{R}(i)$.

It should be clear that these conditions can be verified in polynomial space. In particular, condition 2 can be verified by calling a satisfiability checking algorithm for the concept $\Pi_{C \in r_{x}(i)} \bar{C}$ w.r.t. the TBox $\overline{\mathcal{T}}$, which can be done in NP [1, $2]$. We assume that at every step of the algorithm presented below these conditions are checked after a quasimodel state is guessed, and if any of them fails the algorithm terminates with the negative answer.

Our algorithm works as follows. We guess an initial state $\left\{r_{x}(0) \mid x \in \Delta\right\}$. Then we guess another state $\left\{r_{x}(1) \mid x \in \Delta\right\}$ and check the suitability condition for each pair of types $\left(r_{x}(0), r_{x}(1)\right)$ : for all $C \mathcal{U} D \in e v(\mathcal{K}), C \mathcal{U} D \in r_{x}(0)$ iff either $C \mathcal{U} D, C \in r_{x}(1)$ or $D \in r_{x}(1)$ (if it fails the algorithm answers 'no'). Then, for all $R \in \operatorname{role}^{ \pm}(\mathcal{K})$, we check: if $\exists R=r_{x}(1)$, for some $x$, then $i_{R^{-}} \geq 1$ (if not, the algorithm terminates with the negative answer). After that we erase the previous state $\left\{r_{x}(0) \mid x \in \Delta\right\}$.

We continue in such a fashion until we reach step $K$. From now on the check on roles is different: for all $R \in \operatorname{role}^{ \pm}(\mathcal{K})$, if $\exists R \in r_{x}(K)$ then $i_{R^{-}} \geq K$. After checking the suitability condition we erase the state $K-1$ of the quasimodel and guess $\left\{r_{x}(K+1) \mid x \in \Delta\right\}$. Then we verify: for all $R \in \operatorname{role}^{ \pm}(\mathcal{K})$, if $\exists R \in r_{x}(K+1)$ then $i_{R^{-}} \geq K$. We never erase $\left\{r_{x}(K) \mid x \in \Delta\right\}$ from the memory.

We proceed in this way until we reach step $L^{\prime}$. (At step $K_{0}$ we need to verify whether $\left\{r_{x}\left(K_{0}\right) \mid x \in \Delta\right\}$ is equal to $\left.\left\{r_{x}(K) \mid x \in \Delta\right\}\right)$. We perform all the same checks as at the previous steps. Now, for every $x$, we store the set $\mathrm{ev}_{x}=\left\{D \in \operatorname{cl}(\mathcal{K}) \mid C \mathcal{U} D \in r_{x}\left(L^{\prime}\right)\right\}$. After that we guess $\left\{r_{x}\left(L^{\prime}+1\right) \mid x \in \Delta\right\}$ and perform the same checks as before. If $D \in r_{x}\left(L^{\prime}+1\right)$ and $D \in \mathrm{ev}_{x}$ then we update $\mathrm{ev}_{x}=\mathrm{ev}_{x} \backslash\{D\}$. Next we guess $\left\{r_{x}\left(L^{\prime}+2\right) \mid x \in \Delta\right\}$ and perform the same checks and the same update of $\mathrm{ev}_{x}$ as before. Then erase $\left\{r_{x}\left(L^{\prime}+1\right) \mid x \in \Delta\right\}$. We proceed like that until step $P$. Now, the algorithm answers 'yes' if ev $\mathrm{v}_{x}=\emptyset$ and $r_{x}\left(L^{\prime}\right)=r_{x}(P)$ for all $x \in \Delta$.

Soundness of the presented algorithm follows from Theorem 1 and completeness from Theorems 1 and 2. It is easy to see that the algorithm runs in polynomial space. 


\section{Decision procedure for $T_{\diamond}^{0} D L-L i t e_{b o o l}^{\mathcal{N}}$}

Our algorithm for checking satisfiability of $T_{\diamond}^{0} D L$-Lite $\mathrm{N}_{\text {bool }}^{\mathcal{N}} \mathrm{KB} \mathcal{K}=(\mathcal{T}, \mathcal{A})$ essentially guesses the 'prefix' of length $L+1$ and the period of length $P$ of an ultimately periodical quasimodel $\mathfrak{Q}^{\prime}=\left\langle W^{\prime}, K, L\right\rangle$ for $\mathcal{K}$ as in Theorem 5 , and then checks whether conditions (runs), (stuttr), (obj) in Section 4 hold and whether the types in positions $L+1$ and $L+P+1$ of the prefix coincide for every run.

More precisely, first we guess and store some numbers $L, K$ and $P$ such that $L \leq N_{\mathcal{K}}+|\operatorname{ev}(\mathcal{K})| \cdot\left(\left|\operatorname{role}^{ \pm}(\mathcal{K})\right|+2\right)+3, K \leq L$ and $P \leq|\operatorname{ev}(\mathcal{K})|$. Then we guess a set $\Omega \subseteq \operatorname{role}^{ \pm}(\mathcal{K})$ and numbers $\left\{i_{R} \mid R \in \bar{\Omega}\right\}$ such that $i_{R}<L$, for each $R \in \Omega$. For every $R \in \Omega$, we then guess a sequence $r_{R}$ of length $L+P+2$ of types for $\mathcal{K}$ and, for every $a \in o b(\mathcal{A})$, a sequence $r_{a}$ of length $L+P+2$ of types for $\mathcal{K}$.

Let $W_{0}=\left\{\left(r_{R},\left\{i_{R}\right\}\right) \mid R \in \Omega\right\} \cup\left\{\left(r_{a}, \emptyset\right) \mid a \in o b(\mathcal{A})\right\}$. The set $W_{0}$ can be regarded as a finite representation of the witnesses $W^{\prime}$ from $\mathfrak{Q}^{\prime}$. Now we check that the following conditions hold:

1. $r(K)$ and the $r(i)$, for $L \leq i \leq L+P+1$, are stutter-invariant for each $(r, \Xi) \in W_{0}$

2. if $\bigcirc^{n} B(a) \in \mathcal{A}$ then $B \in r_{a}(n)$; if $\square B(a) \in \mathcal{A}$ then $B \in r_{a}(i)$, for all $0<i \leq L+P+1$

3. for all $i \leq L+P+1$ and $R \in \operatorname{role}^{ \pm}(\mathcal{K})$, if $\exists R^{-} \in r(i)$, for some $(r, \Xi) \in W_{0}$, then $\left(r_{R},\left\{i_{R}\right\}\right) \in W_{0}, \exists R \in r_{R}\left(i_{R}\right)$ and either $i \leq i_{R}<K$ or $K \leq i_{R}<L$;

4. $r(L+1)=r(L+P+1)$, for all $(r, \Xi) \in W_{0}$;

5. $r(i)$ is realisable, for all $(r, \Xi) \in W_{0}$ and $i \leq L+P+1$;

6. for all $(r, \Xi) \in W_{0}, i \leq L+P+1$ and $\diamond C \in r(i)$,

- if $i \leq L$ then there is $j, i<j \leq L+P+1$, such that $C \in r(j)$;

- if $L<i \leq L+P+1$ then there is $j, L<j \leq L+P+1$, such that $C \in r(j)$;

7. for all $(r, \Xi) \in W_{0}, \diamond C \in e v(\mathcal{K})$ and all $i \leq L+P+1$, if $C \in r(i)$ then $\diamond C \in r(j)$, for all $j<i$.

The algorithm returns 'yes' iff all the conditions above are satisfied.

The presented algorithm is sound: indeed, if conditions 1-7 are satisfied we can construct an ultimately periodical quasimodel for $\mathcal{K}$ which, by Theorem 4 , means that $\mathcal{K}$ is satisfiable. The algorithm is also complete: if $\mathcal{K}$ is satisfiable then, by Theorems 4 and 5 , there exists an ultimately periodical quasimodel $\mathfrak{Q}=\left\langle W^{\prime}, K, L\right\rangle$ with period $P$ and $K, L, P$ bounded by polynomial functions in $|\mathcal{K}|$ as above; then $W_{0}$ consisting of the prefixes of length $L+P+2$ of runs in $W^{\prime}$ satisfies conditions $1-7$ and thus the algorithm returns 'yes.' Finally, it is easy to see that $L, K, P$ and $W_{0}$ can be constructed and conditions 1-7 checked by a non-deterministic algorithm that runs in time polynomial in $|\mathcal{K}|$. 